\title{
Development of a non-causal wave energy control algorithm based on artificial intelligence
}

\author{
Li L. ${ }^{1, *}$, Gao Y.. ${ }^{1,2}$, Ning, D.Z. ${ }^{3}$, Yuan, Z.M. ${ }^{1}$ \\ ${ }^{1}$ Department of Naval Architecture, Ocean and Marine Engineering, University of \\ Strathclyde, Glasgow G4 OLZ, UK \\ ${ }^{2}$ State Key Laboratory of Hydraulic Engineering Simulation and Safety, Tianjin \\ University, Tianjin 300072, China \\ ${ }^{3}$ State Key Laboratory of Coastal and Offshore Engineering, Dalian University of \\ Technology, Dalian 116024, China \\ *liang.li@strath.ac.uk
}

\begin{abstract}
The real-time implementation of wave energy control leads to non-causality as the wave load that comes in the next few seconds is used to optimize the control command. The present work tackles non-causality through online forecasting of future wave force using artificial intelligence technique. The past free surface elevation is used to forecast the incoming wave load. A feedforward artificial neural network is developed for the forecasting, which learns to establish the intrinsic link between past free surface elevation and future wave force through machine learning algorithm. With the implementation of the developed online wave force prediction algorithm, a real-time discrete control algorithm taking constraint on response amplitude into account, is developed and implemented to a bi-oscillator wave energy converter in the present research. The dynamic response and the wave power extraction are simulated using a state-space hydrodynamic model. It is shown that the developed real-time control algorithm enhances the power capture substantially whereas the motion of the system is hardly increased. The prediction error effect on power extraction is investigated. The reduction of power extraction is mainly caused by phase error, whilst the amplitude error has minimal influence. A link between the power capture efficiency and the constraint on control is also identified.
\end{abstract}

\section{Highlights}

A real-time declutching wave energy control based on model predictive control strategy is developed.

Artificial intelligence is used to tackle the non-causality. 
Forecasting error effect on control efficiency is investigated.

Constraint effect on control efficiency is investigated.

Keywords: wave energy control; non-causality; wave force prediction; artificial intelligence; power extraction; control constraint.

\section{Nomenclature}

\begin{tabular}{ll}
\hline$\mu(\omega)$ & Frequency-dependent added mass \\
$\lambda(\omega)$ & Frequency-dependent potential damping \\
$\mu_{\infty}$ & Added mass at infinite frequency \\
$h(t)$ & Retardation function \\
$\boldsymbol{A}, \boldsymbol{B}, \boldsymbol{C}$ & System matrices \\
$n$ & Order of the state-space representation \\
$\boldsymbol{u}$ & State vector \\
$H$ & Hamiltonian \\
$\lambda$ & Lagrange multiplier \\
$\beta$ & Control command \\
\hline
\end{tabular}

Word count: 7100

\section{Introduction}

Global renewable power capacity is anticipated to grow by $50 \%$ until 2024 [1]. Such rapid growth is powered by national long-term energy strategies proposed globally. The UK has passed its law aiming at zero net emission by 2050 [2]. The binding renewable energy target for the EU is of at least $32 \%$ of the total energy consumption by 2030 and zero net emission by 2050 [3]. To achieve the long-term zero net emission goal, a diverse renewable energy resource is crucial. The merits of wave energy include high energy density, full-day availability and grid-balancing energy system benefits, etc. Moreover, it has applications for remote islands, powering offshore industries such as fish farms, and oil \& gas platforms. Therefore, the ocean wave energy has been deemed as a high-quality renewable resource of power for an extended period. It is anticipated that wave energy is able to contribute $15 \%$ of the UK's current electricity demand and to have $22 \mathrm{GW}$ installed capacity by 2050 [4]. Various categories of wave energy conversion mechanisms have been developed [5, 6], e.g. the pressure difference [7], the overtopping [8] and the oscillation [9]. However, the wave power capture efficiency is still low, which is one of the obstacles to the large-scale employment of wave energy technology. Innovative techniques enabling the enhancement of 
wave energy efficiency have been identified as an essential route to zero net emission by reducing the energy cost [10].

Wave energy control theory is thus developed to enhance the power harvesting performance of wave energy converter (WEC). The main idea of wave energy control is to tune the response phase of the WEC in order to get resonance manually. Falnes [11] investigated the reactive control intensively, which tunes the response phase by controlling the power take-off (PTO) force. Considering that implementation of reactive control requires the PTO system to return power back to the oscillator during parts of the oscillation cycles, practical implementation of the reactive control remains challenging. Another well-developed control algorithm is the latching control proposed by Budal and Falnes [12], which has been employed widely by researchers $[13,14]$. The latching control tunes the response phase by locking and releasing the oscillator alternately. Once the WEC is released, the buoy velocity would be in phase with the wave load resulting in the enhancement of power capture. Nevertheless, the alternate locking and releasing may lead to fatigue damage. A dual of the latching control is the declutching control [15], which works by loading and offloading the PTO system from the WEC alternately. Compared with the latching control, declutching control is much more convenient to be implemented in practice, e.g., using a simple by-pass valve. A disadvantage of declutching control is that the WEC excursion amplitude will be increased, and in some cases, it is necessary to impose a constraint on the excursion. Babarit et al. [16] implemented the declutching control to the SEAREV (Système Électrique Autonome de Récupération d'Énergie des Vagues) concept, and they showed that the energy production was increased substantially. Similar works have been reported by Feng and Kerrigan [18]. More recently, new optimal wave energy control algorithms have emerged. Zhang and Li [19] proposed the sliding mode control. Zhan et al. [20] developed the so-called economic feedback model predictive control.

Despite the well-established optimal wave energy control theories, the practical implementation of optimal wave energy control is still challenging. One of the obstacles is the non-causality of optimal wave energy control, which indicates that control command is optimized based on future wave excitation force coming in the next few seconds. If the wave energy control is to be implemented in practice, the non-causality must be addressed. There are two primary categories of methodologies to tackle the non-causality. The first category is to make some modifications to the optimal wave energy control algorithm so that it becomes causal. However, the modified control algorithm is sub-optimal, which means that the control efficiency is harmed. Fusco and Ringwood [21] modified the ideal complex conjugate control 
and proposed a causal control, which was sub-optimal. Li and Gao [22] developed a similar causal control algorithm and interpreted in detail how the modified control algorithm became sub-optimal. The second solution group is to forecast the future wave force and optimize the control command based on the predicted future wave force. In the present research, the second group of solutions to non-causality is adopted.

Wave force forecasting is essentially a regression problem. Serval approaches have been proposed and applied, e.g., the autoregressive model, the grey model, the extended Kalman filter, etc. The autoregressive approach was employed by Li et al. [23] to implement the wave force forecasting using. $\mathrm{Li}$ et al. [24] forecasted the incoming irregular ocean waves using the grey model. One limitation of the classic forecasting approaches is that prediction of one variable is based on its past value. In real condition, however, it is not easy to measure the wave force directly, which is the integral of wave pressure across the WEC surface.

The present study aims to propose a feasible wave force forecasting approach and embed it to the non-causal wave energy control to increase the power extraction of WECs. The explosive development of artificial intelligence technology presents an alternative approach to forecast future wave load. The artificial intelligence technology is able to establish the correlation between two variables so that the prediction of future wave load is not necessarily based on its value in the past, but on other measurable variables. In the present research, an online forecasting model is proposed to predict future wave force by monitoring free surface elevation.

The organization of the rest paper is highlighted here. Firstly, the development of the WEC dynamic model is presented in Section 2. Subsequently, Section 3 develops a noncausal model predictive control algorithm for the real-time implementation of wave energy control. The artificial intelligence is then utilized in Section 4 for the prediction of incoming wave force to tackle the non-causality. The numerical model is validated in Section 5. Section 6 investigates the efficiency of the proposed smart control algorithm. Effects of prediction error and control constraint are demonstrated. Eventually, conclusions drawn from the present research are given.

\section{Numerical model}

\subsection{Wave energy converter}

A bi-oscillator WEC is considered in the present research. As illustrated in Fig. 1, the submerged part of the buoy is a hemisphere with a diameter of $10 \mathrm{~m}$, and the upside is a 
cylinder with the same radius. To concentrate on the scope of the present research, the buoy only oscillates up and down. The internal oscillator slides along the vertical axis of the buoy, which is connected to the buoy through a linear spring. The PTO system converts the relative motion between the two oscillators into energy. In this study, the PTO system is represented by a linear damper.

Two coordinates are defined. Coordinate 1 is a global one fixed to the ground. The coordinate origin is at the undisturbed free surface level with the positive $\mathrm{Z}$ axis upward. Coordinate 2 is attached to and moves together with the buoy. The coordinate origin is at the equilibrium position of the internal oscillator, and the positive $\mathrm{Z}$ axis is upward as well.

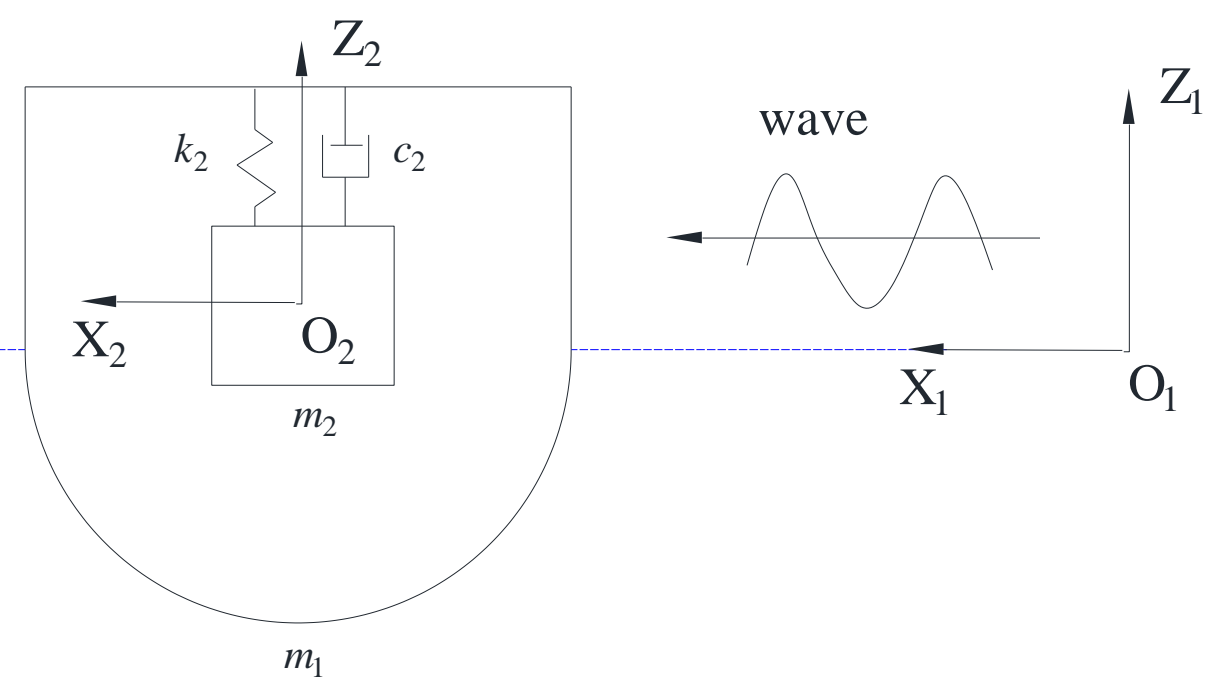

Fig. 1. Wave energy converter.

\subsection{Motion equation}

According to the Cummins' equation [25], the motion equation of the bi-oscillator is given by

$$
\begin{aligned}
& \left(m_{1}+\mu_{\infty}\right) \ddot{z}_{1}+m_{2}\left(\ddot{z}_{1}+\ddot{z}_{2}\right)+\int_{0}^{t} h(t-\tau) \dot{z}_{1}(\tau) d \tau+d_{1} \dot{z}_{1}+k_{1} z_{1}=F_{\text {wave }} \\
& m_{2}\left(\ddot{z}_{1}+\ddot{z}_{2}\right)+d_{2} \dot{z}_{2}+k_{2} z_{2}=0
\end{aligned}
$$

with:

- $\mathrm{z}_{1}$ the displacement of the buoy relative to the ground (defined in the global coordinate), $\mathrm{z}_{2}$ the displacement of the internal oscillator relative to the buoy (defined in the body-fixed coordinate)

- $m_{i}$ the mass of the body i. $m_{1}=1.88 \times 10^{5} \mathrm{~kg}, m_{2}=8.05 \times 10^{5} \mathrm{~kg}$, so that the total weight is equal to the buoyance.

- $\mu_{\infty}$ the hydrodynamic added mass of the buoy at infinite frequency 
- $k_{1}=789.7 \mathrm{kN} / \mathrm{m}$ the hydrostatic restoring stiffness of the buoy; $d_{1}=50.4 \mathrm{kNs} / \mathrm{m}$ the additional linear damping representing the viscous load on the external buoy. $d_{1}$ is equal to $5 \%$ critical damping.

- $\quad k_{2}$ the spring connecting the external buoy and the internal oscillator; $d_{2}$ the damping coefficient of the PTO system. $k_{2}=94.8 \mathrm{kN} / \mathrm{m}$ and $d_{2}=50 \mathrm{kNs} / \mathrm{m}$ are employed so as the natural period of the internal oscillator (about $6.3 \mathrm{~s}$ ) is close to wave peak period that is to be considered in the present research.

- $h(t)$ is the so-called retardation kernel function, representing the memory effect of the free surface. It can be obtained from the potential damping $\lambda(\omega)$

$$
h(t)=\frac{2}{\pi} \int_{0}^{\infty} \lambda(\omega) \cos (\omega t) d \omega
$$

- $F_{\text {wave }}(t)$ the wave excitation force. In the present research, the time series of wave excitation force is generated by

$$
\begin{aligned}
& F_{\text {wave }}(t)=\operatorname{Re}\left\{\sum_{i=1}^{N} \psi\left(\omega_{i}\right) \cdot \eta\left(\omega_{i}\right) \cdot e^{i\left(\omega_{i} t+\varepsilon_{i}\right)}\right\} \\
& \eta\left(\omega_{i}\right)=\sqrt{2 S\left(\omega_{i}\right) \Delta \omega}
\end{aligned}
$$

with $\Psi(\omega)$ being the transfer function of linear wave excitation force. $S(\omega)$ is the JONSWAP wave spectrum, representing the power distribution of the irregular ocean waves. $\varepsilon$ is a random value between $[0,2 \pi]$ representing the random phase difference between wave components of various oscillating frequencies.

The boundary element analysis software Wadam [26] is used to calculate the hydrodynamic quantities listed above. To focus on the scope of the present research, the linear potential flow has been applied whereas other modelling approaches are also available. Zabala et al. [27] investigated the advantages and disadvantages of existing tools and proposed an integrated modelling framework, namely the so-called 'Digital prototype'. Penalba [28] presented a review on mathematical modelling of wave energy converters, with an emphasis on the nonlinear approaches.

However, it is inconvenient to implement the real-time control strategy with Eq. (1) and thus a state-space model is developed, in which the convolution term is replaced by a statespace representation. Denoting $f(t)$ the radiation force, the following formulas are an approximation of each other

$$
f(t)=\int_{0}^{t} h(t-\tau) \dot{z}_{1}(\tau) \mathrm{d} \tau
$$




$$
\begin{aligned}
\frac{\mathrm{d}^{n} f}{\mathrm{~d} t^{n}}+q_{n-1} \frac{\mathrm{d}^{n-1} f}{\mathrm{~d} t^{n-1}}+\ldots+q_{1} \frac{\mathrm{d} f}{\mathrm{~d} t}+q_{0} f & =p_{n-1} \frac{\mathrm{d}^{n-1} \dot{z}_{1}}{\mathrm{~d} t^{n-1}}+p_{n-2} \frac{\mathrm{d}^{n-2} \dot{z}_{1}}{\mathrm{~d} t^{n-2}}+\ldots+p_{1} \frac{\mathrm{d} \dot{z}_{1}}{\mathrm{~d} t}+p_{0} \dot{z}_{1} \\
f(t) & =\boldsymbol{C} \cdot \boldsymbol{u}(t) \\
\dot{\boldsymbol{u}}(t) & =\boldsymbol{A} \cdot \boldsymbol{u}(t)+\boldsymbol{B} \dot{z}_{1}(t)
\end{aligned}
$$

where $\boldsymbol{A}, \boldsymbol{B}$ and $\boldsymbol{C}$ are system matrices. Their dimensions are $n \times n, n \times 1$ and $1 \times n . n$ is the order of the system. $n=5$ is employed in the current hydrodynamic model. $\boldsymbol{u}$ can be regarded as a type of intermediate vector.

Combing Eq. (5) and Eq. (6)

$$
\begin{aligned}
\boldsymbol{A} & =\left[\begin{array}{ccccc}
-q_{n-1} & -q_{n-1} & \cdots & -q_{1} & -q_{0} \\
1 & 0 & \cdots & 0 & 0 \\
0 & 1 & \cdots & 0 & 0 \\
\vdots & \vdots & \ddots & 0 & 0 \\
0 & 0 & \cdots & 1 & 0
\end{array}\right], \boldsymbol{B}=\left[\begin{array}{c}
1 \\
0 \\
0 \\
\vdots \\
0
\end{array}\right] \\
\boldsymbol{C} & =\left[\begin{array}{lllll}
p_{n-1} & p_{n-2} & \cdots & p_{1} & p_{0}
\end{array}\right]
\end{aligned}
$$

Combing Eq. (4) and Eq. (6)

$$
h(t)=\boldsymbol{C} e^{A t} \boldsymbol{B}
$$

Taking the Laplace transform of Eq. (5), one gets

$$
h(s)=\frac{Y(s)}{U(s)}=\frac{p_{n-1} s^{n-1}+p_{n-2} s^{n-2}+\cdots+p_{0}}{s^{n}+q_{n-1} s^{n-1}+\cdots+q_{0}}
$$

Eq. (2) is the time-domain expression of retardation kernel function, and it can be transformed to the frequency-domain through the Fourier transformation

$$
h(i \omega)=\int_{0}^{\infty} h(\tau) e^{-i \omega \tau} d t \tau=\lambda(\omega)+i \omega\left[\mu(\omega)-\mu_{\infty}\right]
$$

Eq. (8) and Eq. (9) are correlated through the Fourier transformation

$$
\tilde{F}\{h(t)\}=\left.h(s)\right|_{s=i \omega}=h(i \omega)
$$

According to Eq. (11)

$$
\frac{p_{n-1}(i \omega)^{n-1}+p_{n-2}(i \omega)^{n-2}+\ldots+p_{0}}{(i \omega)^{n}+q_{n-1}(i \omega)^{n-1}+\ldots+q_{0}}=\lambda(\omega)+i \omega\left[\mu(\omega)-\mu_{\infty}\right]
$$

so that

$$
\begin{aligned}
& \lambda(\omega)=\operatorname{Re}\left\{\frac{p_{n-1}(i \omega)^{n-1}+p_{n-2}(i \omega)^{n-2}+\ldots+p_{1}(i \omega)^{1}+p_{0}}{(i \omega)^{n}+q_{n-1}(i \omega)^{n-1}+\ldots+q_{1}(i \omega)^{1}+q_{0}}\right\} \\
& \mu(\omega)=\operatorname{Im}\left\{\frac{1}{\omega} \cdot \frac{p_{n-1}(i \omega)^{n-1}+p_{n-2}(i \omega)^{n-2}+\ldots+p_{1}(i \omega)^{1}+p_{0}}{(i \omega)^{n}+q_{n-1}(i \omega)^{n-1}+\ldots+q_{1}(i \omega)^{1}+q_{0}}\right\}+\mu_{\infty}
\end{aligned}
$$


Parameters $\boldsymbol{p}$ and $\boldsymbol{q}$ are estimated by the least square method, known as system identification. Fig. 2 gives the system identification system result with $n=5$. As shown, a fifth-order state-space model is a good representation and thus $n=5$ is employed hereinafter.
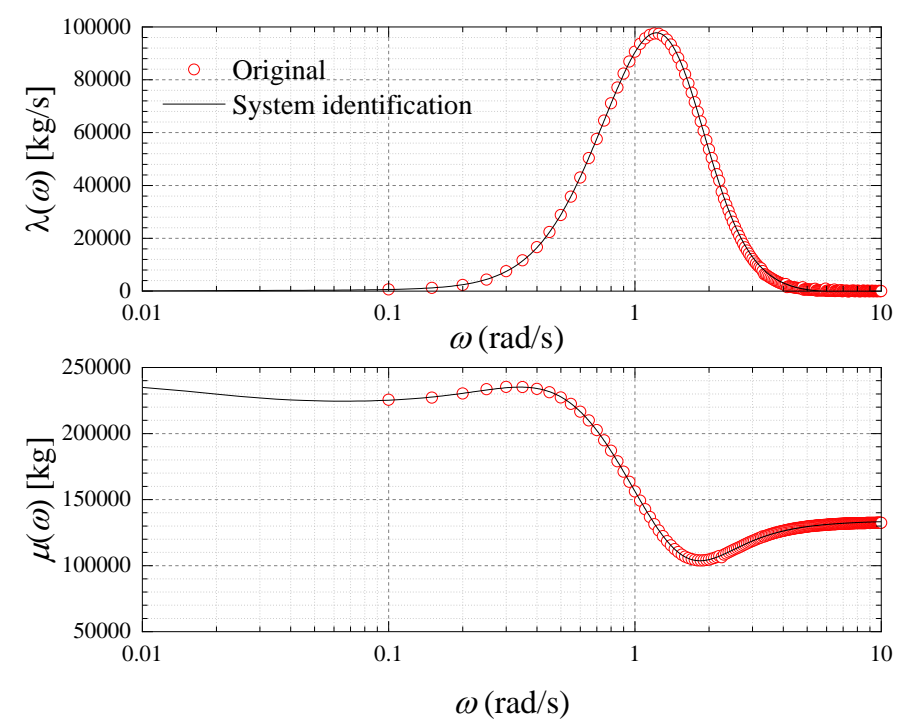

Fig. 2. System identification result, $n=5$.

By using the state-space representation, Eq. (1) can be re-written as

$$
\begin{aligned}
& \left(m_{1}+\mu_{\infty}\right) \ddot{z}_{1}+m_{2}\left(\ddot{z}_{1}+\ddot{z}_{2}\right)+\boldsymbol{C} \boldsymbol{u}+d_{1} \dot{z}_{1}+k_{1} z_{1}=F_{\text {wave }} \\
& m_{2}\left(\ddot{z}_{1}+\ddot{z}_{2}\right)+d_{2} \dot{z}_{2}+k_{2} z_{2}=0 \\
& \dot{\boldsymbol{u}}=\boldsymbol{A} \boldsymbol{u}+\boldsymbol{B} \dot{z}_{1}
\end{aligned}
$$

Denote a state vector $\boldsymbol{X}=\left[z_{1}, z_{2}, \dot{z}_{1}, \dot{z}_{2}, \boldsymbol{u}^{T}\right]^{T}$. Then, Eq. (14) is transformed into a firstorder differential equation

$$
\begin{aligned}
& \dot{\boldsymbol{X}}=\boldsymbol{\gamma} \cdot \boldsymbol{X}+\boldsymbol{\kappa} \\
& \boldsymbol{\gamma}=\left[\begin{array}{ccccc}
0 & 0 & 1 & 0 & \mathbf{0} \\
0 & 0 & 0 & 1 & \mathbf{0} \\
-\frac{k_{1}}{m_{1}+\mu_{\infty}} & \frac{k_{2}}{m_{1}+\mu_{\infty}} & -\frac{d_{1}}{m_{1}+\mu_{\infty}} & \frac{d_{2}}{m_{1}+\mu_{\infty}} & -\frac{\boldsymbol{C}}{m_{1}+\mu_{\infty}} \\
\frac{k_{1}}{m_{1}+\mu_{\infty}} & -\frac{\left(m_{1}+m_{2}+\mu_{\infty}\right) k_{2}}{\left(m_{1}+\mu_{\infty}\right) m_{2}} & \frac{d_{1}}{m_{1}+\mu_{\infty}} & -\frac{\left(m_{1}+m_{2}+\mu_{\infty}\right) d_{2}}{\left(m_{1}+\mu_{\infty}\right) m_{2}} & \frac{\boldsymbol{C}}{m_{1}+\mu_{\infty}} \\
\mathbf{0} & \mathbf{0} & \boldsymbol{B} & \mathbf{0} & \boldsymbol{A}
\end{array}\right], \boldsymbol{\kappa}=\left[\begin{array}{c}
0 \\
0 \\
\frac{F_{\text {wave }}}{m_{1}+\mu_{\infty}} \\
-\frac{F_{\text {wave }}}{m_{1}+\mu_{\infty}} \\
\mathbf{0}
\end{array}\right]
\end{aligned}
$$

With the pre-given initial condition $\boldsymbol{X}(0)=\mathbf{0}$, Eq. (15) is solved as a classical initial value problem following the $4^{\text {th }}$ Runge-Kutta algorithm. The power extraction of the bi-oscillator WEC is

$$
P=\frac{1}{T} \int_{0}^{T} d_{2} \cdot \dot{z}_{2}(t)^{2} d t
$$




\section{Real-time declutching control algorithm}

\subsection{Optimal declutching control}

In the present research, declutching control is used to increase the power extraction by connecting and disconnecting the PTO system alternately according to the control command $\beta(t)$. When the PTO system is loaded, $\beta=1$ and $\beta=0$ not. The switch between loading and offloading could be realized using a by-pass valve for a hydraulic PTO or a power-electronic switch if the PTO is a direct generator. Declutching control is non-causal since the future wave force must be known to derive the control command. At this point, it is assumed that the future wave force is known whilst the next section will interpret how the future wave force is forecasted in the present research.

With the implementation of declutching control, Eq. (15) can be re-expressed as $\dot{\boldsymbol{X}}=\boldsymbol{\gamma} \cdot \boldsymbol{X}+\boldsymbol{\kappa}$

$$
\boldsymbol{\gamma}=\left[\begin{array}{ccccc}
0 & 0 & 1 & 0 & \mathbf{0} \\
0 & 0 & 0 & 1 & \mathbf{0} \\
-\frac{k_{1}}{m_{1}+\mu_{\infty}} & \frac{k_{2}}{m_{1}+\mu_{\infty}} & -\frac{d_{1}}{m_{1}+\mu_{\infty}} & \frac{\beta d_{2}}{m_{1}+\mu_{\infty}} & -\frac{\boldsymbol{C}}{m_{1}+\mu_{\infty}} \\
\frac{k_{1}}{m_{1}+\mu_{\infty}} & -\frac{\left(m_{1}+m_{2}+\mu_{\infty}\right) k_{2}}{\left(m_{1}+\mu_{\infty}\right) m_{2}} & \frac{d_{1}}{m_{1}+\mu_{\infty}} & -\frac{\beta\left(m_{1}+m_{2}+\mu_{\infty}\right) d_{2}}{\left(m_{1}+\mu_{\infty}\right) m_{2}} & \frac{\boldsymbol{C}}{m_{1}+\mu_{\infty}} \\
\mathbf{0} & \mathbf{0} & \boldsymbol{B} & \mathbf{0} & \boldsymbol{A}
\end{array}\right], \boldsymbol{\kappa}=\left[\begin{array}{c}
0 \\
0 \\
\frac{F_{\text {wave }}}{m_{1}+\mu_{\infty}} \\
-\frac{F_{\text {wave }}}{m_{1}+\mu_{\infty}} \\
\mathbf{0}
\end{array}\right]
$$

As shown in Eq. (17), the dynamics of the bi-oscillator is not continuous as $\beta$ varies abruptly between 0 and 1, implying that the system switches between two different states (PTO loaded and unloaded). It is worth noting that a delay exists when a practical controller decides to apply the declutching action. The lag effect was numerically modelled by Clément and Babarit [29]. It is not the scope of the present study, and thereby the lag effect is neglected.

Wave energy control is a constrained optimization problem from a mathematic point of view, namely the optimization of the objective function $P$ with respect to the control command $\beta$ in the presence of constraint Eq. (17). Multiple optimization algorithms are available, e.g., the quadratic programming and the Lagrange multiplier method. In the present research, the Lagrange multiplier method is employed

$$
H=\beta d_{2} \dot{z}_{2}^{2}+\lambda \cdot(\gamma \cdot \boldsymbol{X}+\boldsymbol{\kappa})
$$


where $\lambda$ is the Lagrange multiplier and $H$ is the Hamiltonian. Through the introduction of the Lagrange multiplier, the original constrained optimization is converted into an unconstrained problem. Please note that the single variable $\beta$ switches between 0 and 1 , so Eq. (18) is optimized when

$$
\beta=\left\{\begin{array}{cc}
1 \quad \dot{z}_{2}^{2}+\frac{\lambda_{3}}{m_{1}+\mu} \dot{z}_{2}-\frac{\lambda_{4}\left(m_{1}+m_{2}+\mu\right)}{\left(m_{1}+\mu\right) m_{2}} \dot{z}_{2}>0 \\
0 \quad \text { otherwise }
\end{array}\right.
$$

Now, it is to set up an equation to describe $\lambda$. According to Babarit et al. [16]:

$$
\dot{\lambda}_{i}=-\frac{\partial H}{\partial X_{i}}(t, X, \beta)
$$

Although Eq. (20) is also a differential formula, it can not be addressed as an initial value problem as $\lambda(0)$ must be in accordance with the control algorithm [30]. Borne et al. [30] proposed a final condition $\lambda(T)=\mathbf{0}$ and got the Lagrange multiplier with an iterative process (see Fig. 3). The same approach is employed to optimize the control command. Firstly, launch the simulation with $\beta(t)=0$ to obtain the dynamic response free of control by integrating Eq. (17) from $t=0$ to $t=T$. $\lambda$ is subsequently obtained by integrating Eq. (20) from $t=T$ to $t=0(\lambda(T)=\mathbf{0}$ is now an initial condition). Based on Eq. (19), the control command $\beta(t)$ is derived. Re-launch the simulation with the derived control command to update the dynamic response. Iterate the process to optimize the control command until it converges. 


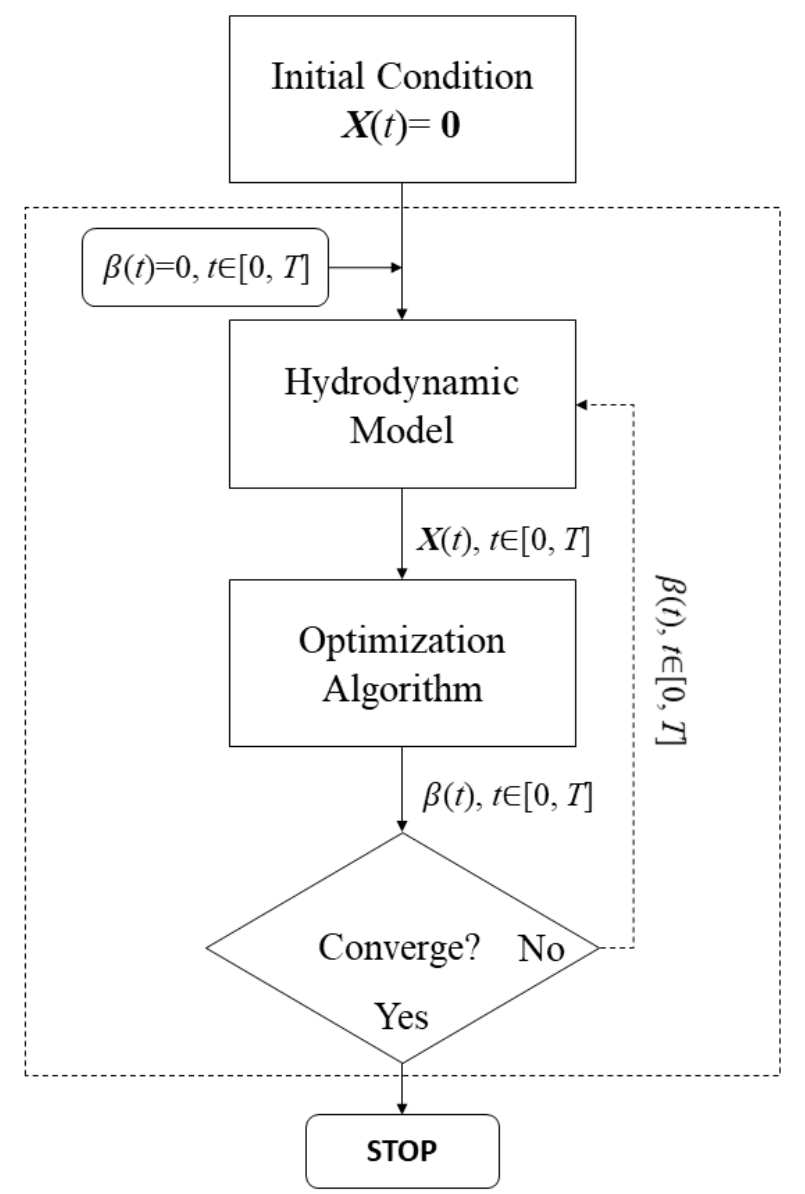

Fig. 3 Process of optimal declutching control algorithm.

Fig. 4 plots the velocity of the inner oscillator under regular wave. As shown, the PTO system is switched on and off alternately according to the optimized control command. Considering that the PTO force is offloaded occasionally, the inner oscillator is subject to less resistance so that its oscillation is amplified. Once the PTO system is loaded again, it experiences a much stronger relative motion between the two oscillators resulting in the enhancement of power capture.

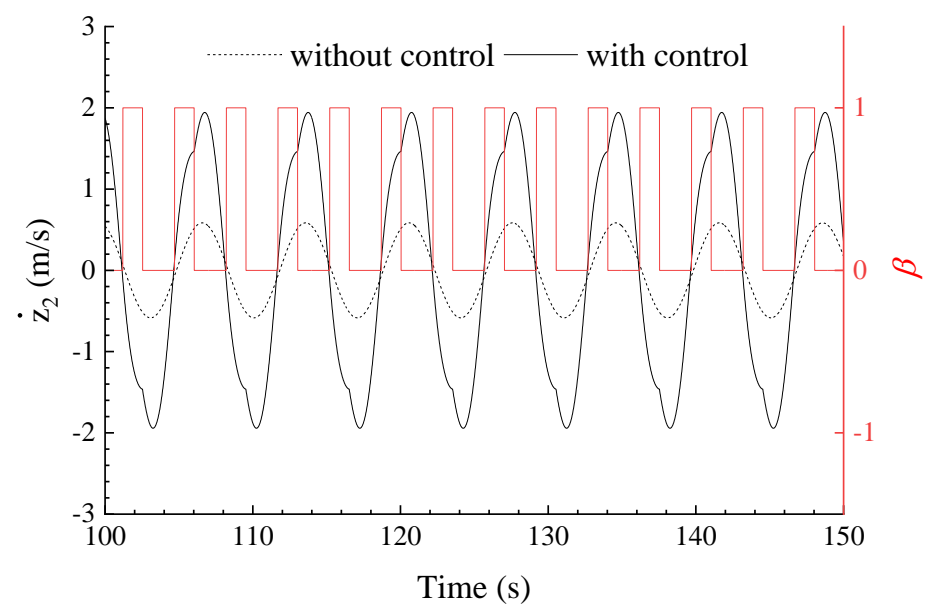

Fig. 4. Time series of inner oscillator velocity under regular wave (wave height is $1 \mathrm{~m}$, wave period is $7 \mathrm{~s}$ ). 


\subsection{Model predictive control strategy}

Optimal declutching control utilizes wave load over the entire time interval $[0, T]$ to maximize the power extraction. Although various types of forecasting modelling exist, the prediction is only short-term whereas the future wave force over the entire interval $[0, T]$ is unpredictable. It indicates that optimal declutching control cannot be implemented directly. To tackle this problem, a modified declutching control based on the model predictive control strategy is employed. The model predictive control strategy has been confirmed effective in the implementation of other wave energy control algorithms [13, 31, 32]. Here, we employ the model predictive control strategy to implement real-time declutching control.

The model predictive strategy optimizes the control command over a finite and short time interval $\left[t_{i}, t_{i}+\Delta t\right]$ rather than optimizing Eq. (18) over the entire time interval $[0, T]$. Therefore, it is optimal over time interval $\left[t_{i}, t_{i}+\Delta t\right]$ but sub-optimal over entire time interval $[0, T]$. The control command is optimized at each time instant over the updated time interval $\left[t_{i}, t_{i}+\Delta t\right]$, and thereby the declutching control is implemented online.

Similarly, the control command over time horizon $\left[t_{i}, t_{i}+\Delta t\right]$ is optimized using the iterative procedure as illustrated in Fig. 3, in which an initial control command $\beta(t)=0$ is assumed. It is effective, but not efficient as the control command is optimized only after a certain amount of iterations. Wu et al. [33] suggested that control command optimized at previous time step can be used as the initial control command at the current time step. To further enhance the computation efficiency, they executed the iteration only once. Here, the same approach is adopted (see Table 1).

Table 1 Modified model predictive control strategy $(N$ represents the simulation length).
\begin{tabular}{|l|l|}
\hline Standard model predictive control strategy & $\begin{array}{l}\text { Modified model predictive control strategy } \\
{[33]}\end{array}$ \\
\hline for $i=2: N$ & for $i=2: N$ \\
\hline Predict the future wave force over $\left[t_{i}, t_{i+p}\right]$ & Predict the future wave force over $\left[t_{i}, t_{i+p}\right]$ \\
\hline for $j=1: \infty$ & for $j=1: 1$ \\
\hline Assume an initial command $\beta(t)=0$ & Adopt command $\beta(t)$ at previous step $t_{i-1}$ \\
\hline Optimize the control command $\beta(t)$ & Optimize the control command $\beta(t)$ \\
\hline end, if $\beta(t)$ converges & end \\
\hline Implement the control according to command & $\begin{array}{l}\text { Implement the control according to command } \\
u\left(t_{i}\right)\end{array}$ \\
\hline end & end \\
\hline
\end{tabular}

\subsection{Constraint of control}

The previous section develops the declutching control algorithm without restrictions. In real conditions, however, a limit must be imposed on the excursion of the PTO system. Thus, 
there is an upper bound on the relative displacement between the two oscillators. A braking strategy is used to implement an amplitude constraint to the WEC. Whenever the relative displacement $z_{2}$ exceeds a threshold $Z_{0}$, a large damping force is applied to lock the internal oscillator. Thus, the dynamics of the bi-oscillator with constraint is further expressed as

$$
\begin{aligned}
& \dot{\boldsymbol{X}}=\boldsymbol{\gamma} \cdot \boldsymbol{X}+\boldsymbol{\kappa} \\
& \boldsymbol{\gamma}=\left[\begin{array}{ccccc}
0 & 0 & 1 & 0 & \mathbf{0} \\
0 & 0 & 0 & 1 & \mathbf{0} \\
-\frac{k_{1}}{m_{1}+\mu} & \frac{k_{2}}{m_{1}+\mu} & -\frac{d_{1}}{m_{1}+\mu} & \frac{\beta d_{2}+\alpha d_{3}}{m_{1}+\mu} & -\frac{\boldsymbol{C}}{m_{1}+\mu} \\
\frac{k_{1}}{m_{1}+\mu} & -\frac{\left(m_{1}+m_{2}+\mu\right) k_{2}}{\left(m_{1}+\mu\right) m_{2}} & \frac{d_{1}}{m_{1}+\mu} & -\frac{\beta\left(m_{1}+m_{2}+\mu\right) d_{2}+\alpha d_{3}}{\left(m_{1}+\mu\right) m_{2}} & \frac{\boldsymbol{C}}{m_{1}+\mu} \\
\mathbf{0} & \mathbf{0} & \boldsymbol{B} & \mathbf{0} & \boldsymbol{A}
\end{array}\right], \boldsymbol{\kappa}=\left[\begin{array}{c}
0 \\
0 \\
\frac{F_{\text {wave }}}{m_{1}+\mu} \\
-\frac{\boldsymbol{F}_{\text {wave }}}{m_{1}+\mu} \\
\mathbf{0}
\end{array}\right] \\
& \alpha=\left\{\begin{array}{l}
1, z_{2} \geq Z_{0} \\
0, z_{2}<Z_{0}
\end{array}\right.
\end{aligned}
$$

where $d_{3}$ is a finite and huge damping coefficient.

\section{Online wave force forecasting}

The present work forecasts future wave force by measuring the wave elevations online. At each time instant, the free surface elevations over the past 5 seconds are inputted into a neural network to forecast the wave load that comes in the next 5 seconds. The sampling rate is $0.1 \mathrm{~s}$, so that 50 data points of past wave elevation are used to forecast 50 future wave force data points. The forecasting process is updated online in accordance with the model predictive control strategy presented in Section 3.2.

\section{1. forecasting algorithm}

A feedforward backpropagation neural network with six layers (one input layer, four hidden layers and one output layer), as shown in Fig. 5, is employed to forecast the future wave force. There are 10 neurons in each layer. The network is trained to establish the intrinsic link between past free surface elevation and future wave force. Once this link has been established, future wave force could be forecasted based on past free surface elevation. 


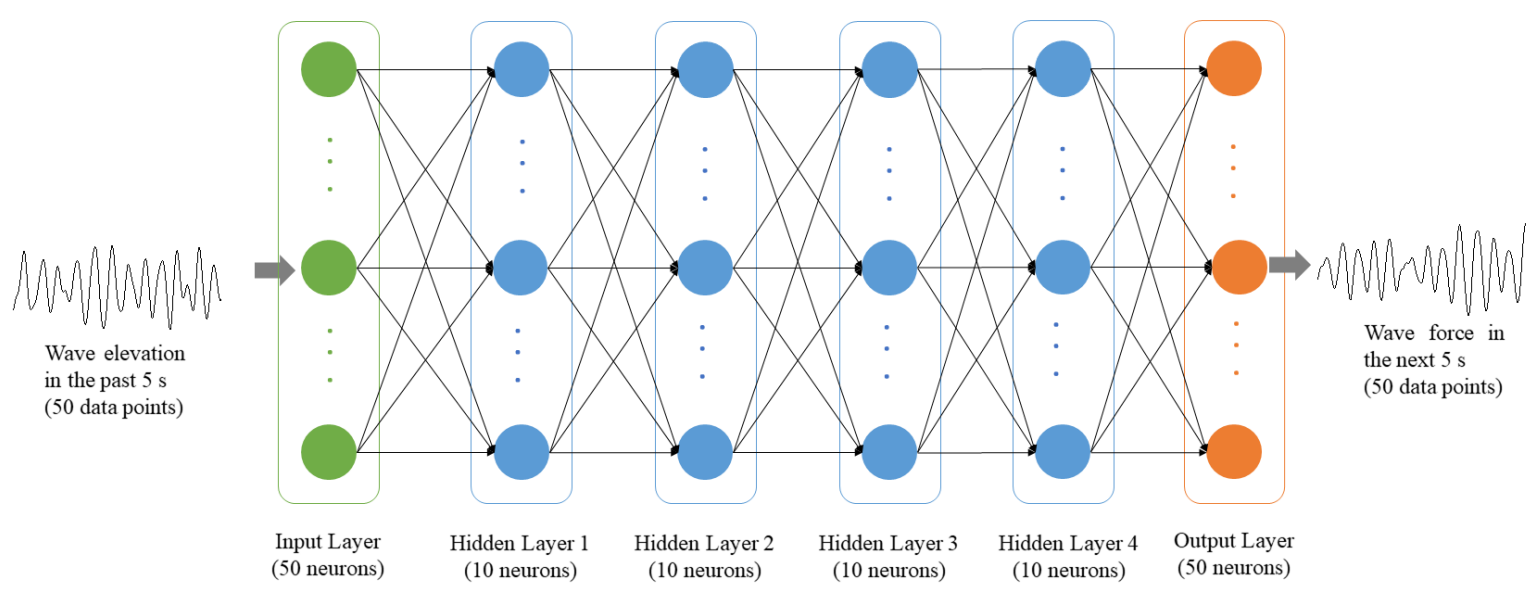

Fig. 5. Feedforward backpropagation artificial neural network.

The machine learning algorithm is used to establish such link with a large amount of examples. The learning data are generated manually by Eq. (3). From a mathematic point of view, the nature of the so-called 'training' is to optimize parameters $\boldsymbol{w}$ and $\boldsymbol{b}$ so as to minimize the forecasting error $\Theta$

$$
\Theta(\boldsymbol{w}, \boldsymbol{b})=\frac{1}{2 L} \sum_{k=1}^{L}\left\|y^{k}-a^{k}\right\|^{2}
$$

$\boldsymbol{w}$ is the weight and $\boldsymbol{b}$ is the bias. $L$ is the number of learning examples. $y^{k}$ is the forecasting output, and $a^{k}$ is the actual value. The gradient descent method and the backpropagation method are used to optimize $\boldsymbol{w}$ and $\boldsymbol{b}$. The training process is based on an iteration basis, which optimizes $\boldsymbol{w}$ and $\boldsymbol{b}$ using the gradient descent method.

$$
\begin{gathered}
w_{j i}{ }^{\prime}=w_{j i}-\kappa \frac{\partial \Theta}{\partial w_{j i}} \\
b_{j i}{ }^{\prime}=b_{j i}-\kappa \frac{\partial \Theta}{\partial b_{j i}}
\end{gathered}
$$

where $w_{j i}{ }^{\prime}$ and $b_{j i}{ }^{\prime}$ are the optimized parameters after one iteration. $\kappa$ is known as the learning rate, a parameter used to represent how 'fast' the network learns during the training process. The gradient of the forecasting error is estimated by the backpropagation approach. Please refer to Refs [34, 35] for more information of the training process.

The significance of the present forecasting approach is that the real-time wave surface elevation, which can be easily measured using various types of techniques, is employed to predict the future wave force. It makes great sense in practical application as the wave force, which is very difficult to measure, is no longer needed in the prediction. The trained neural network is represented by parameters $\boldsymbol{w}$ and $\boldsymbol{b}$, also making it very convenient to implement in practice without extra cost. Moreover, the forecasting method is robust to the wave state as the wave surface elevation, rather than the wave spectra which is available at certain intervals 
and site-dependent, is employed for the forecasting. It enables the forecasting method to be implemented in real-time and employed in a wide range of ocean sites. Therefore, the present wave prediction algorithm can be deemed a feasible and promising solution to the enhancement of wave energy efficiency.

\subsection{Forecasting accuracy}

The accuracy of the incoming wave load prediction is essential to the control efficiency $[24,36]$ as the forecasted future wave load is inputted into the controller while the oscillation is induced by the actual wave force. It is worth investigating the forecasting accuracy before the implementation of the wave energy control. Fig. 6 compares the forecasted wave load and the real situation, with the forecasting length $\Delta t=5 \mathrm{~s}$. Despite the slight discrepancy, which is inevitable, the time series of the forecasted future wave force match the real situation well.

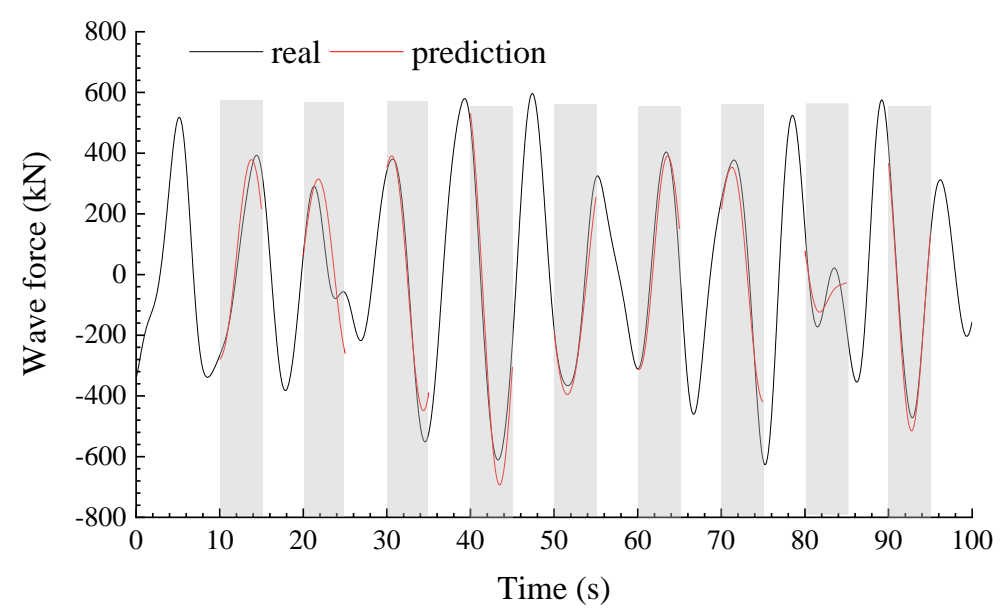

Fig. 6. Prediction performance of the trained artificial neural network.

To further evaluate the forecasting accuracy, a predictability index $R$ [37] is employed

$$
R=\frac{E\left\{F_{\text {wave }}(t+\Delta t \mid t)^{2}\right\}}{E\left\{F_{\text {wave }}(t+\Delta t)^{2}\right\}}
$$

where $F_{\text {wave }}(t+\Delta t \mid t)$ is the forecasted future wave force at time instant $t+\Delta t$, which is predicted $\Delta t$ ahead at time instant $t . F_{\text {wave }}(t+\Delta t)$ is real future wave force at time instant $t+\Delta t$. $R>1$ indicates that the predicted value is larger than the real value. The predictability index $R$ corresponding to the 1 hour period is 1.07 , indicating a good prediction. It implies that the control command could be derived appropriately even if the future wave force is predicted. Section 6.2 will further investigate the forecasting uncertainty effect on the control performance. 


\section{Validation}

The developed numerical model is validated against the analytical result in this section. In regular wave, the analytical dynamic model of the bi-oscillator can be expressed as

$$
\begin{aligned}
& -\omega^{2}\left\{m_{1}+\mu(\omega)\right\} z_{1}-\omega^{2} m_{2}\left(z_{1}+z_{2}\right)+i \omega\left\{\lambda(\omega)+d_{1}\right\}+k_{1} z_{1}=F_{\text {wave }} \\
& -\omega^{2} m_{2}\left(z_{1}+z_{2}\right)+i \omega d_{2} z_{2}+k_{2} z_{2}=0
\end{aligned}
$$

Thus, the analytical dynamic motion of the bi-oscillator is

$$
\left[\begin{array}{c}
z_{1} \\
z_{2}
\end{array}\right]=\left[\begin{array}{cc}
-\omega^{2}\left\{m_{1}+m_{2}+\mu(\omega)\right\}+K_{1}+i \omega\left\{\lambda(\omega)+d_{1}\right\} & -\omega^{2} m_{2} \\
-\omega^{2} m_{2} & -\omega^{2} m_{2}+K_{2}+i \omega d_{2}
\end{array}\right]^{-1} \cdot\left[\begin{array}{c}
F_{\text {wave }} \\
0
\end{array}\right]
$$

Fig. 7 compares the motions of the bi-oscillator obtained with the present time-domain numerical model (Eq. (15)) and the analytical formula (Eq. (26)), respectively. The agreement is quite good and thereby the developed numerical model can be deemed validated.

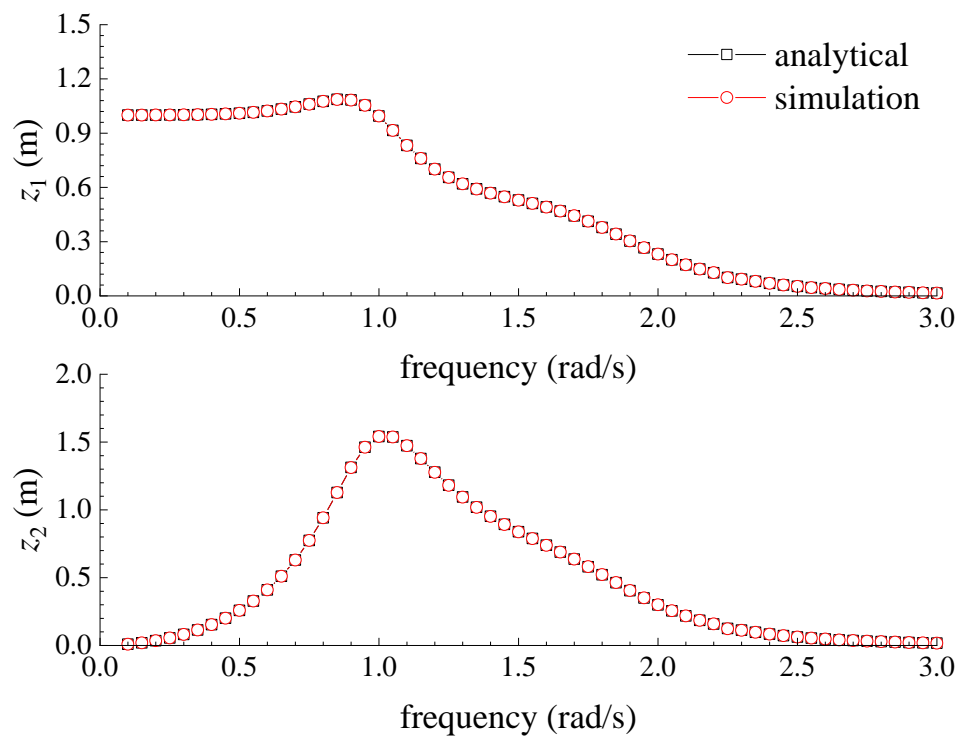

Fig. 7 Validation of the developed time-domain numerical model (wave height $=1 \mathrm{~m})$.

\section{Results and discussions}

The power extraction of the bi-oscillator WEC with and without the proposed real-time declutching control is investigated. The sea state in the East China Sea during summer 2012, which was measured by $\mathrm{Wu}$ et al. [38], is considered. Table 2 summaries the sea wave conditions.

Table 2 Sea states

\begin{tabular}{llll}
\hline & Hs $(\mathrm{m})$ & Tp $(\mathrm{s})$ & Shape factor \\
\hline June & 1.54 & 7.79 & 3.3 \\
July & 1.33 & 6.88 & 3.3 \\
August & 2.39 & 8.18 & 3.3 \\
\hline
\end{tabular}




\subsection{Performance with unconstraint declutching control}

\subsubsection{WEC dynamics}

In real condition, it is expected that the motion excursion of the two oscillators could be limited within a specific range. Significant relative motion is likely to produce undesired structural loads and even exceed the design limits of the power production facilities. In this subsection, the movements of the two oscillators are investigated.

Fig. 8 presents the power spectrum density (PSD) of the buoy motion. As expected, most of the response energy is located around the wave energy frequency range. Additionally, it is found that the control effect on the motion of the external buoy is minimal. It implies that the control is a minor driving factor in the design of the external structure.

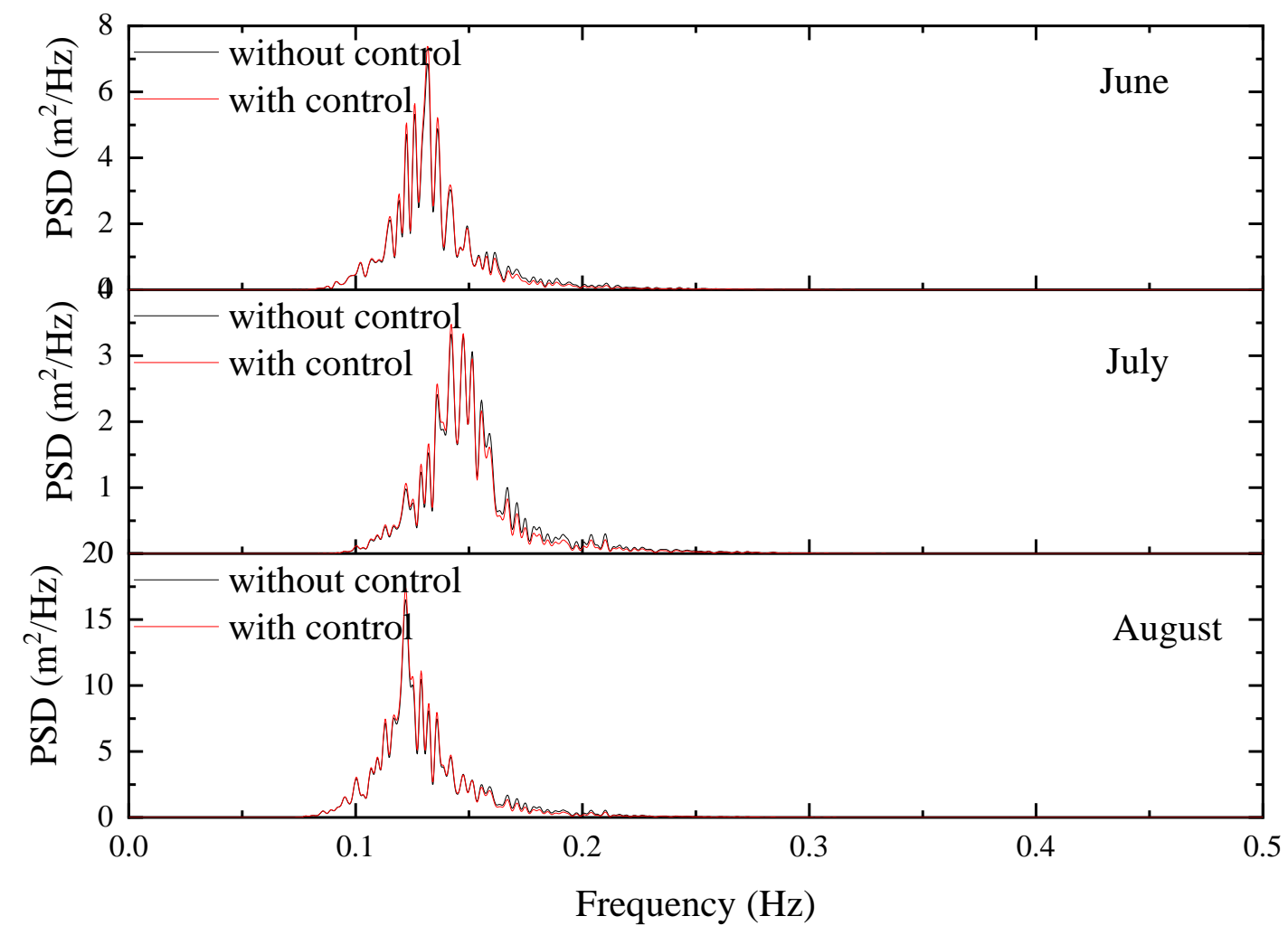

Fig. 8 PSD of the external buoy motion.

Comparatively, the control effect on the internal oscillator motion is noticeable. As presented in Fig. 9, the internal oscillator is stimulated substantially with the implementation of declutching control. From the power extraction point of view, it is desirable since the electric power is converted from the kinetic movement of the internal oscillator. However, large internal motion excursion may exceed the physical allowance of the PTO system, and thus a constraint control algorithm is necessary. Section 6.3 will address this issue. 


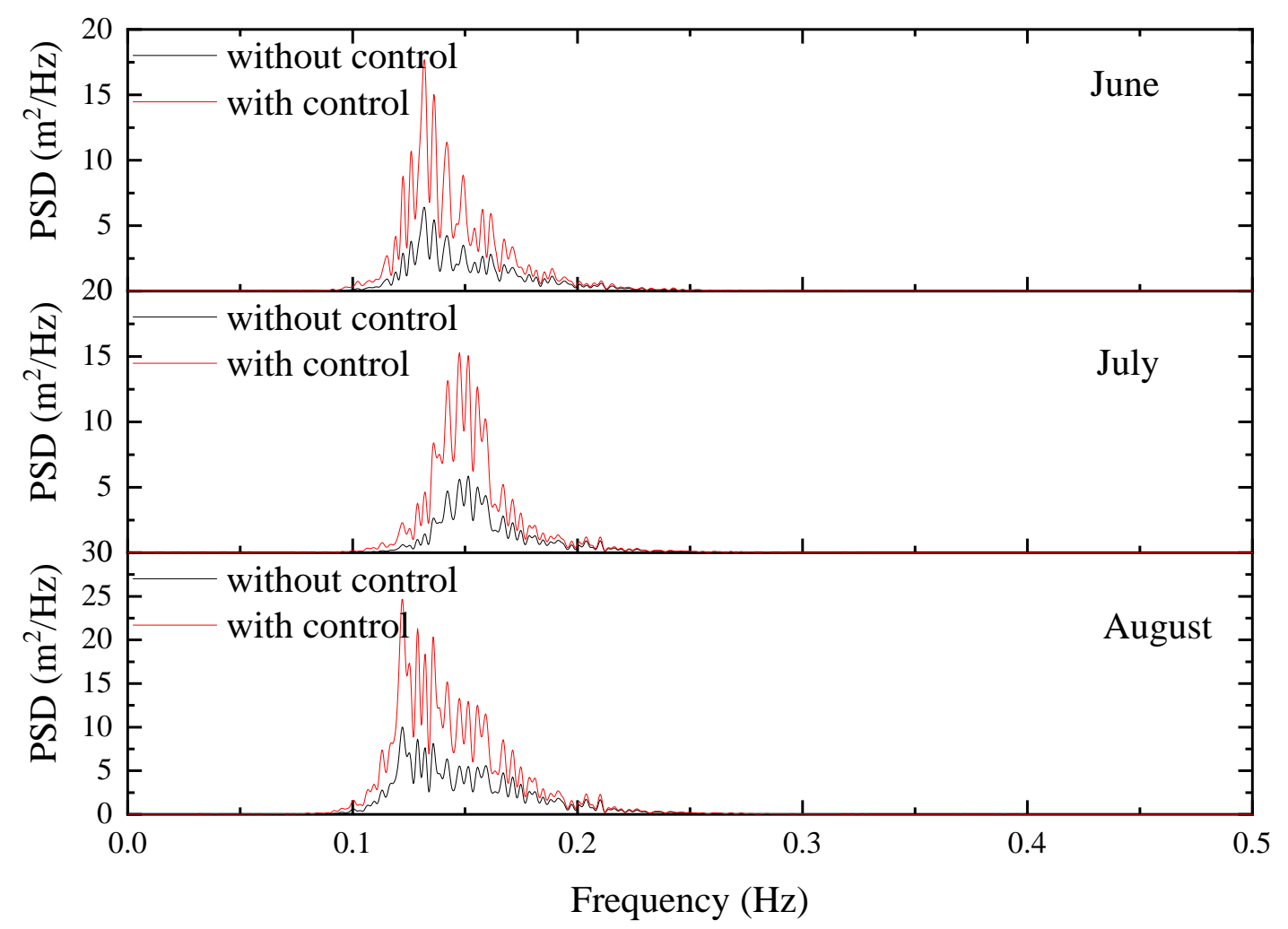

Fig. 9. PSD of internal oscillator motion.

\subsubsection{Power extraction}

Fig. 10 gives the total wave energy absorbed during 1-hour period for the three load cases considered. Since the significant wave height in August is much larger than those in the other two months, the total energy absorption is nearly doubled in August. Regardless of the sea states, one can find that the total energy absorption is increased by around $30 \%$ with implementation of the proposed real-time declutching control.

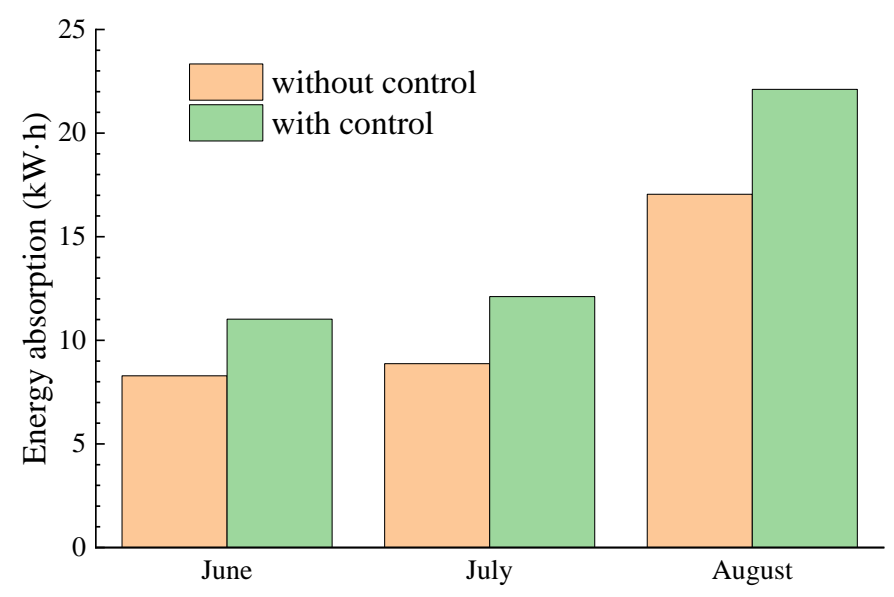

Fig. 10. 1-hr total energy absorption.

To further demonstrate the control effect on the power extraction, its distribution is illustrated in Fig. 11. When the declutching control is not implemented, the majority of wave 
energy is converted when the PTO system operates with relatively low power $(0 \sim 30 \mathrm{~kW})$, accounting to around $70 \%$ of the total generated power. As long as the controller is activated, a reduction of the energy absorption within this range is seen, dropping to $3.95 \mathrm{~kW} \cdot \mathrm{h}$ from $5.85 \mathrm{~kW} \cdot \mathrm{h}$. It is not a surprise since the PTO is offloaded occasionally and no power is generated during the switch-off period. Due to the switching-off of the PTO system, the internal oscillator is subject to fewer resistive loads, leading to the amplification of its oscillation. It indicates that the PTO could operate with high power once it is loaded back again (see Fig. 12). For example, a total of electricity energy of $3.20 \mathrm{~kW} \cdot \mathrm{h}$ is generated within $[30 \mathrm{~kW}, 60 \mathrm{~kW}]$ and $1.72 \mathrm{~kW} \cdot \mathrm{h}$ within $[60 \mathrm{~kW}, 90 \mathrm{~kW}]$. Although the controller disconnects the PTO system occasionally and no power is generated, the PTO system is able to operate with high power so that the total energy absorption is increased. This mechanism is somewhat similar with the complex conjugate control, which feeds energy back to the WEC during parts of the oscillation cycles to accelerate it.

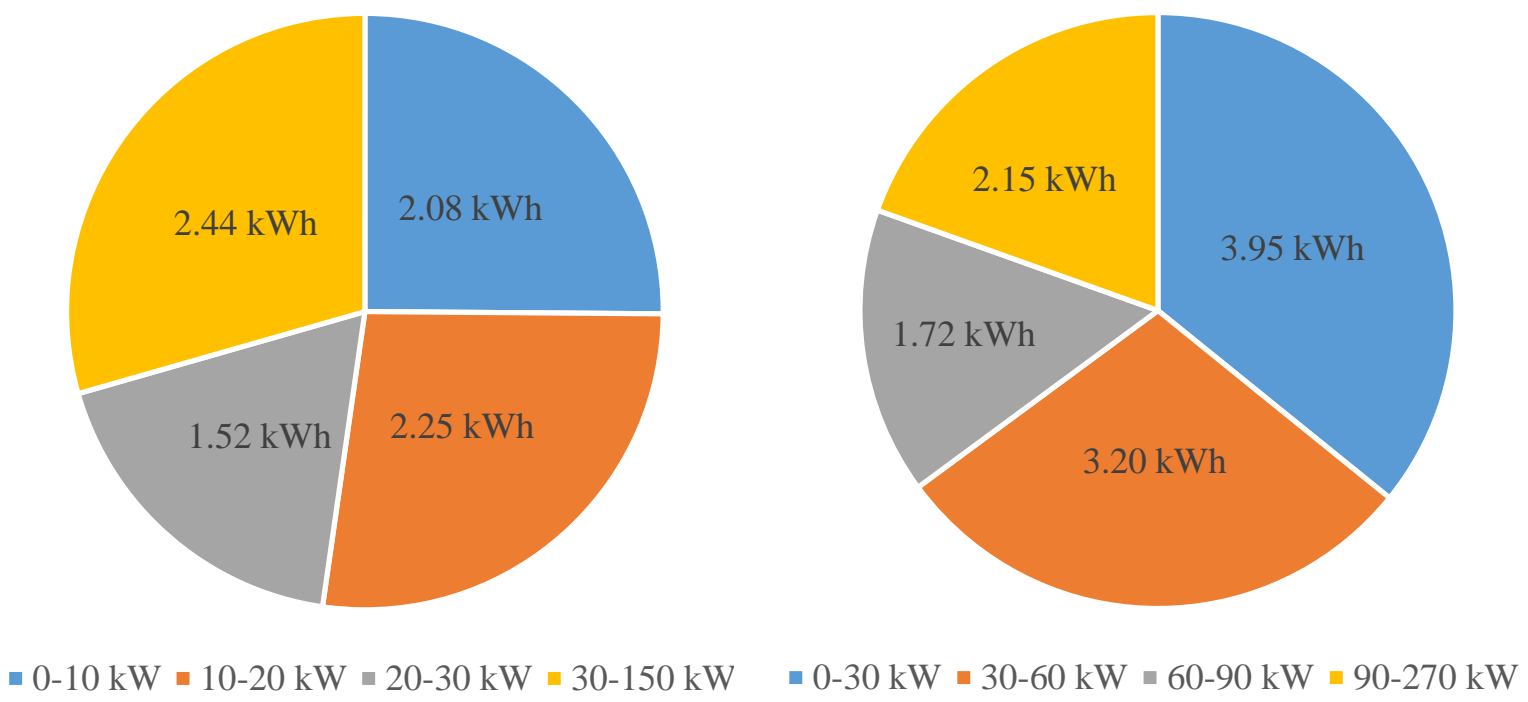

Fig. 11. Power extraction and its distribution, June. Left: without control; Right: with control. 


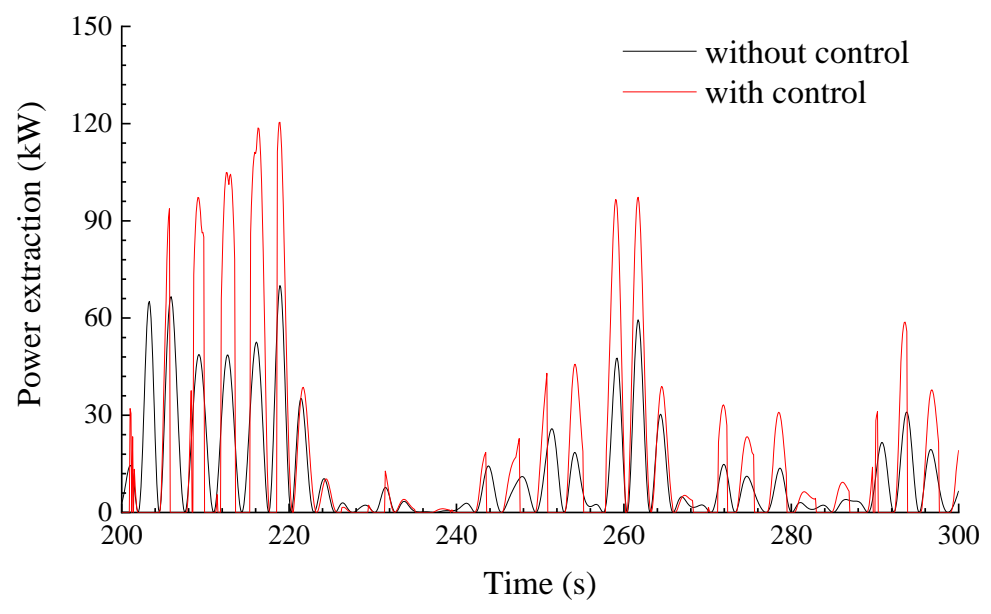

Fig. 12 Time series of instantaneous power capture, June.

\subsubsection{Fatigue load}

It has been confirmed that the proposed declutching control strategy is able to increase the power extraction substantially. In practice, it is also worth an investigation on the control effect on the structural loads, since the average time to structural failure could be affected with the control action. To get a quantitative estimation of the declutching control effect on the fatigue load applied on the PTO system, the load cycle of the PTO system is counted using the rain-flow algorithm, according to the ASTM E1049 standard [39]. The counting is based on 3600 s numerical realization.

Fig. 13 compares the load cycle counts when the WEC operates with and without the declutching control. Generally, the load cycle counts are tripled within the low load amplitude $(0 \sim 50 \mathrm{kN})$ and high load amplitude (100 250 kN) ranges, when the declutching controller is active. The load cycle seems to be insensitive to the control activities within the moderate load range $(50 \sim 100 \mathrm{kN})$. According to the Miner's Rule, the fatigue damage accumulates linearly with the load cycles. Considering that the load cycle counts have been increased by the declutching control, the fatigue damage will accumulate more rapidly and the structure is more likely to fail. It implies that the control effect should be considered carefully in the structural design stage. 


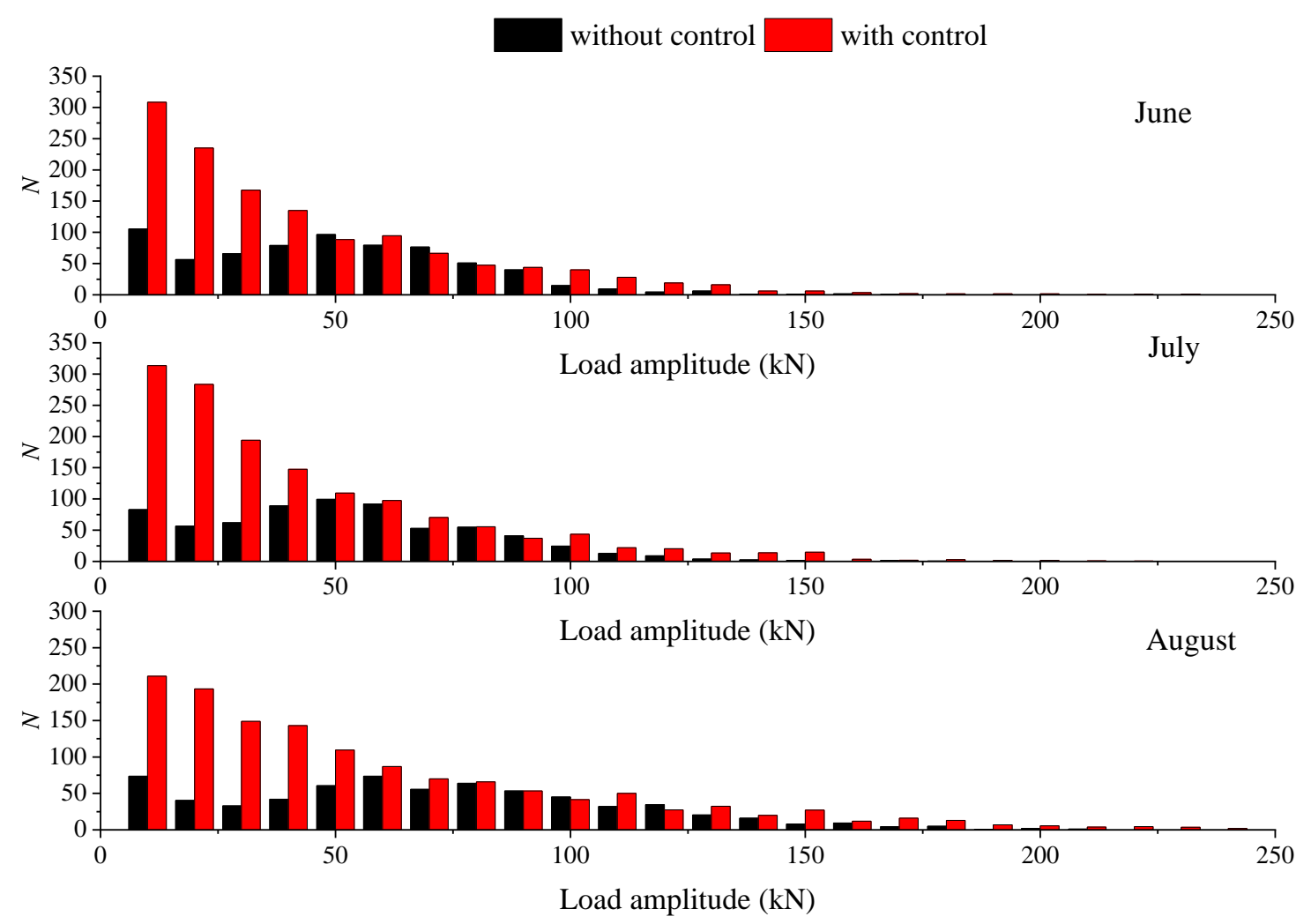

Fig. 13. Load cycle counts of the PTO force.

\subsection{Forecasting effect}

As illustrated in Section 3.2, the proposed real-time declutching control is sub-optimal as the control command is optimized over the forecasting length. Moreover, the forecasted wave force is used to optimize the control command, whereas the oscillation is induced by the actual wave force. This section will investigate how the two factors affect power extraction of the bi-oscillator WEC.

\subsubsection{Forecasting length}

The forecasting length effect is investigated individually by inputting the actual future wave force into the controller manually so as to eliminate the forecasting error. Therefore, the online wave force prediction model is not employed. Fig. 14 shows the effect of forecasting length on the energy absorption. Generally, the WEC always harvest more wave energy when a longer receding horizon length is employed. Three regions are identified, where the energy absorption reacts to the variance of forecasting length in different manners. With the employment of short forecasting length, the declutching control seems to be not useful at all as the power generation is hardly enhanced. Comparatively, the power capture performance is enhance substantially within range $[2 \mathrm{~s}, 7 \mathrm{~s}]$. The energy absorption increases rapidly within the forecasting length. After that, the increasing increment gradually slows down. 
Recall optimization of the control command. The final condition $\lambda(t+\Delta t)=\mathbf{0}$ proposed by Borne et al. [30] is applied to get the control command optimized based on an iteration procedure. It is worth stressing that this so-called final condition is the initial condition used by the control command optimization. As presented previously, the Lagrange multiplier $\lambda$ is derived by integrating Eq. (20) with the initial condition $\lambda(T)=\mathbf{0}$, which induces a transient effect. When the forecasting length $\Delta t$ is sufficiently long, the transient impact will not propagate back to time instant $t$. On the contrary, the control command is not derived correctly due to the transient impact. Although a long horizon length is preferable in order to enhance power extraction, it requires intensive computation in a short time step. Moreover, it is more challenging to predict a longer-term future wave force. Therefore, a moderate horizon length $5 \mathrm{~s}$ is employed in the present research.

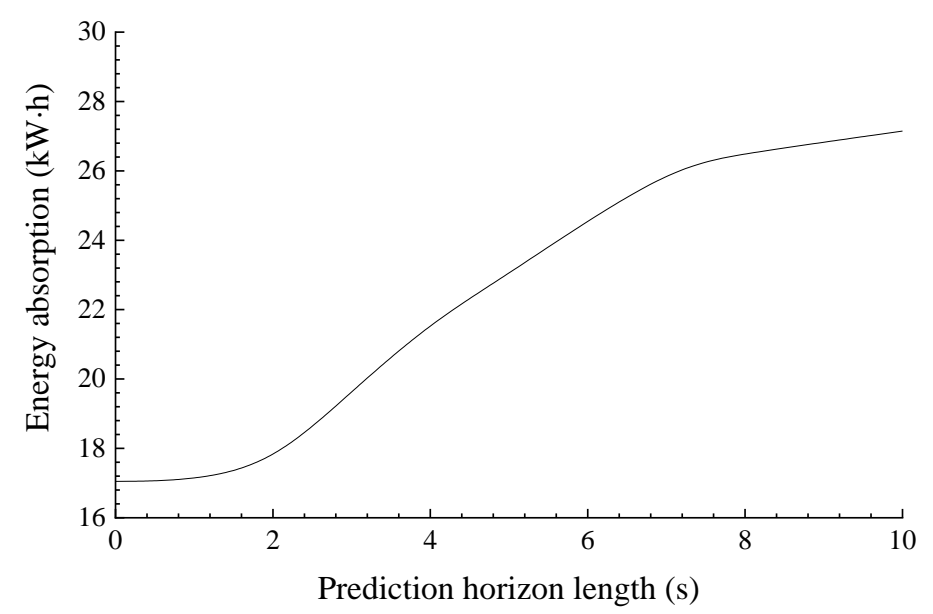

Fig. 14. Effect of receding horizon length on the control efficiency (August).

\subsubsection{Forecasting error}

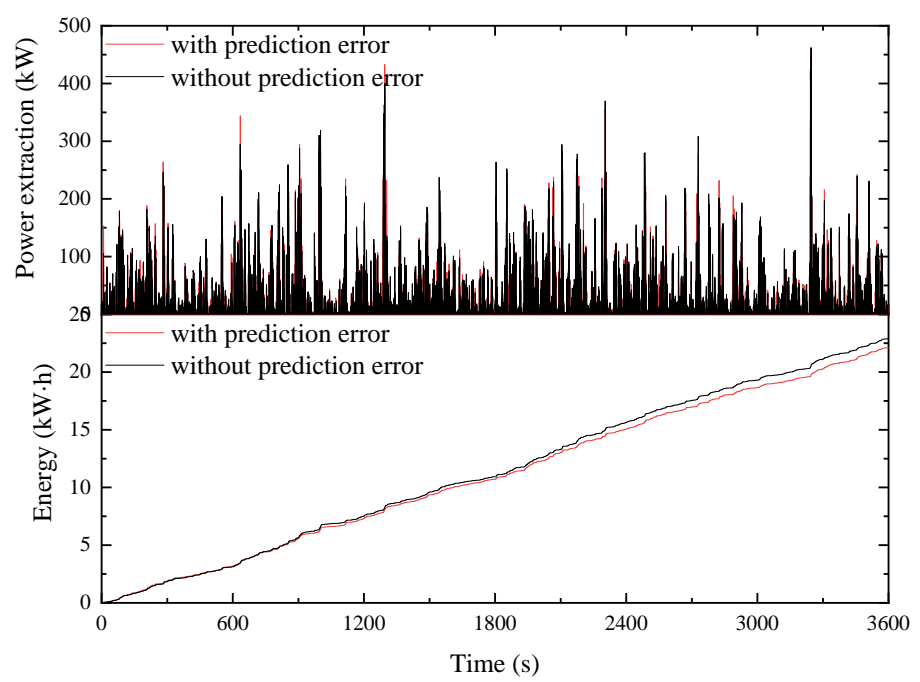

Fig. 15. Effect of forecasting error on the energy harvesting (August).

Fig. 15 demonstrates the effect of forecasting error on the power capturing performance, where the forecasting error leads to a $5 \%$ reduction of the total energy absorption. According 
to Zhang et al. [17], the forecasting error involves two aspects, i.e., the phase error and the amplitude error. To investigate the two parts quantitatively, the present work defines the forecasted future wave force manually

$$
\begin{aligned}
& \bar{F}_{\text {amplitude }}(t)=\operatorname{Re}\left\{\alpha \cdot \psi(\omega) \cdot e^{i \omega t}\right\} \\
& \bar{F}_{\text {phase }}(t)=\operatorname{Re}\left\{\psi(\omega) \cdot e^{i(\omega t+\theta)}\right\}
\end{aligned}
$$

where $\alpha$ represents the amplitude error and $\theta$ represents the phase error.

Fig. 16 illustrates the sensitivity of average power to the prediction error in regular waves. As shown, the amplitude error effect on control efficiency is negligible. Comparatively, the phase error effect on the control efficiency is noticeable. A positive link between energy loss and phase error has been identified. The maximum power is achieved at $\theta=0$, where the prediction error is omitted.
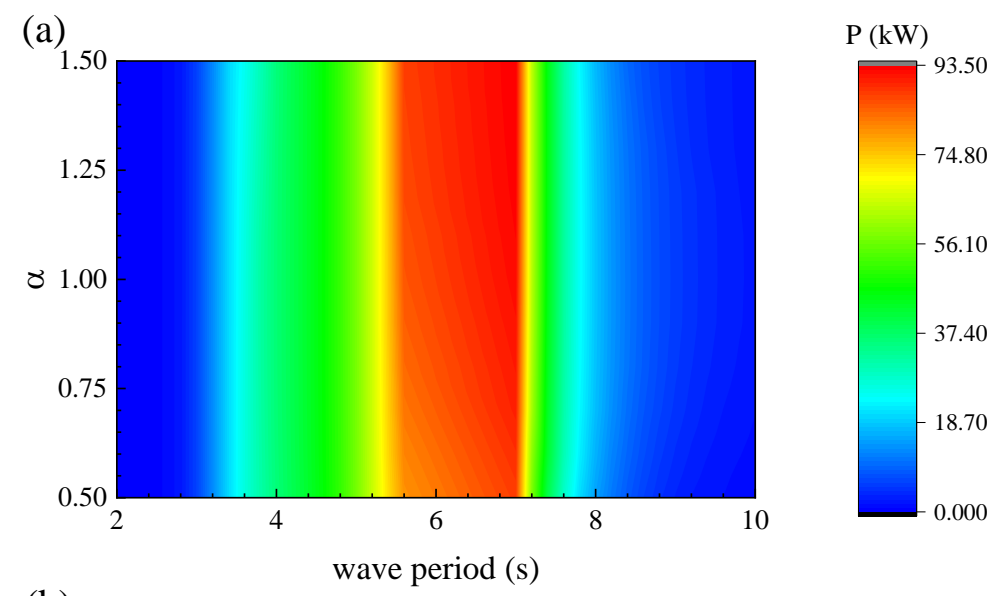

(b)

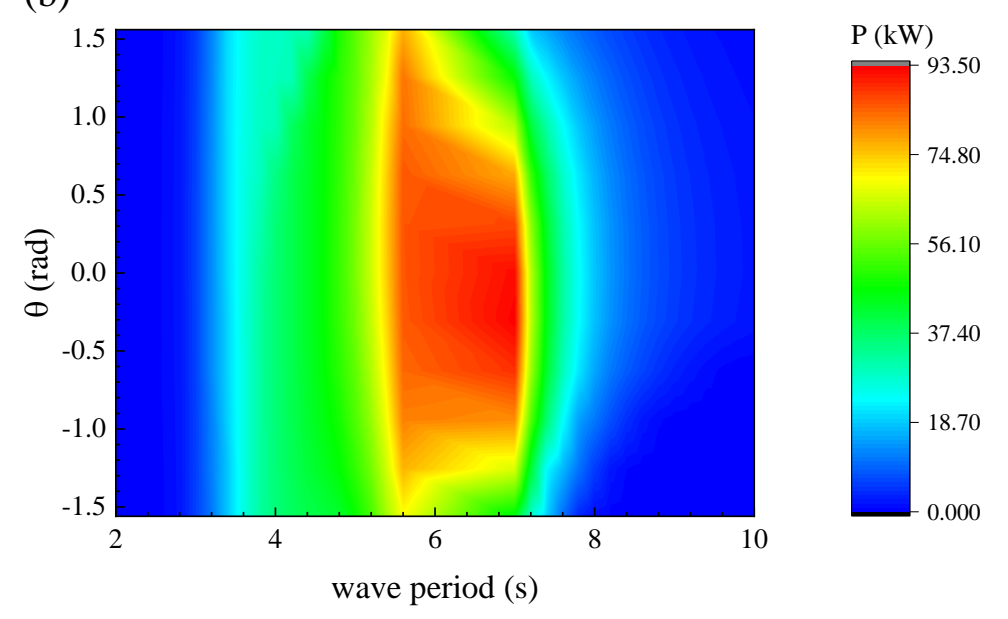

Fig. 16. Prediction error effect on the control efficiency. (a) amplitude error; (b) phase error.

\subsection{Constraint effect}

Due to the implementation of the real-time declutching control, the relative motion between the two oscillators is augmented so as to increase the power extraction. In practice, it is necessary to apply a physical constraint to the relative movement as large excursion may 
cause damage to the PTO system. This section adopts the braking strategy developed in Section 3.3 and implements a constraint to the relative displacement between the two oscillators. Fig. 17 plots the times series of the constrained relative displacement over 1-hour period. It indicates that the proposed constraint strategy is effective.

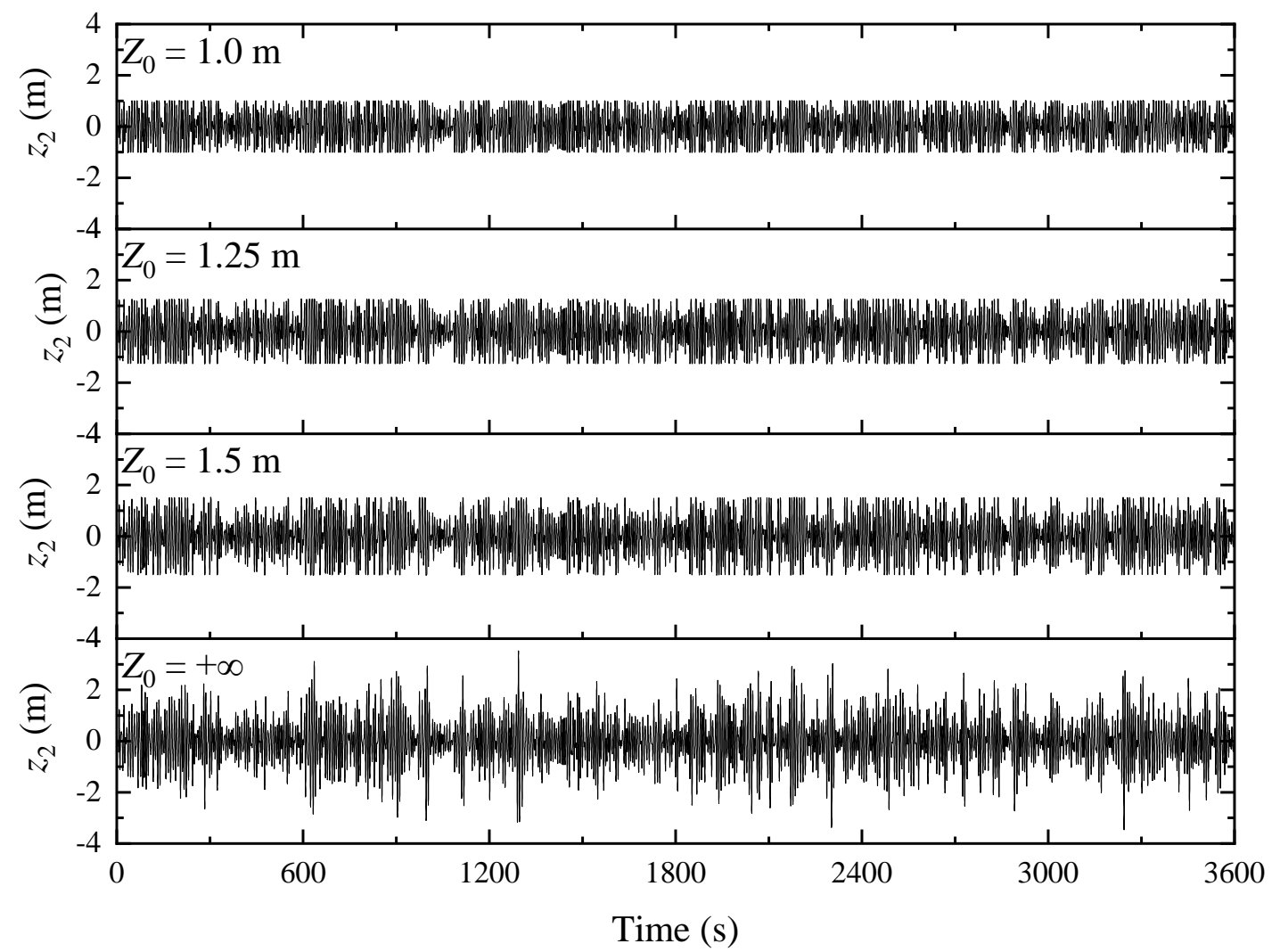

Fig. 17. Relative motion between the two oscillators with constraint (August).

Fig. 18 demonstrates the constraint effect on energy absorption. Whether the declutching control is implemented or not, the total electric energy generated decreases with the constraint. Since the electrical power originates from the relative motion between the two oscillators, it is straightforward that the motion constraint will lead to a reduction of energy capturing. Additionally, the controlled energy absorption is more sensitive to the constraint. When $Z_{0}$ is not very low, the WEC still harvest more energy with the implementation of the declutching control. The energy absorption decreases so rapidly that the WEC captures less power with the declutching control when $Z_{0} \leq 1.25 \mathrm{~m}$. 


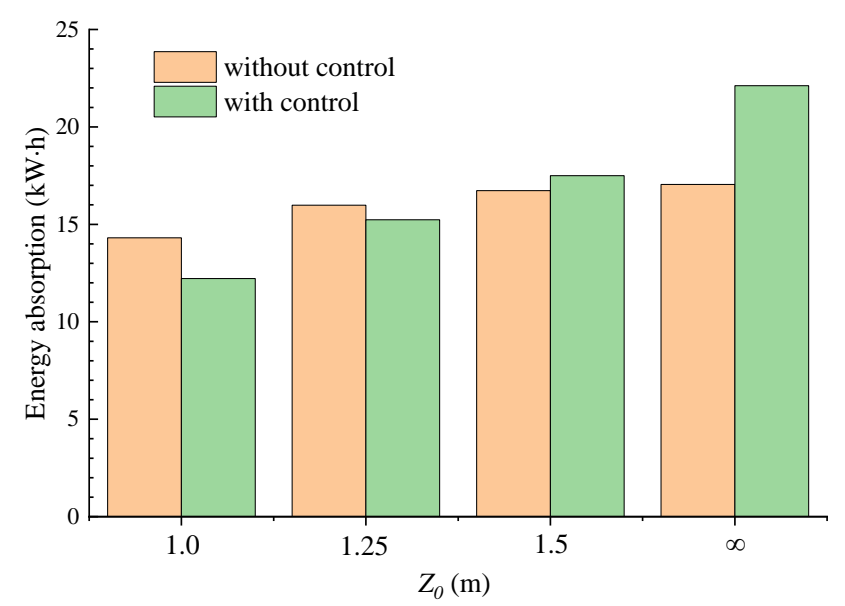

Fig. 18. Effect of constraint on the energy absorption (August).

While the relative motion is restricted, the fatigue load cycle is affected at the same time. Table 3 summarises the load cycle counts with different levels of control constraint. The low amplitude cycle counts increase substantially with low-level constraint. The cycle counts with respect to moderate load amplitude is relatively insensitive to the control constraint. Although the total load cycle counts are increased with the constraint, the counts within high amplitude range are reduced. Since the typical S-N curve of a structure commonly follows the exponential form, it indicates that the structure is much more sensitive to high amplitude fatigue load. Therefore, it can be understood that the PTO system is less likely to fail with a constraint control.

Table 3 Load cycles of PTO force (August).

\begin{tabular}{lllll}
\hline & $Z_{0}=+\infty$ & $Z_{0}=1.5 \mathrm{~m}$ & $Z_{0}=1.25 \mathrm{~m}$ & $Z_{0}=1.0 \mathrm{~m}$ \\
\hline $0-60 \mathrm{kN}$ & 590 & 747 & 831 & 937 \\
$60-120 \mathrm{kN}$ & 294 & 274 & 275 & 273 \\
$120-180 \mathrm{kN}$ & 120 & 120 & 115 & 107 \\
$>180 \mathrm{kN}$ & 43 & 30 & 27 & 13 \\
Total & 1047 & 1171 & 1248 & 1330 \\
\hline
\end{tabular}

Although the dynamics of the internal oscillator is restricted, the external buoy is insensitive to the control constraint. As shown in Fig. 19, the response of the external buoy is hardly varied regardless of the constraint imposed on the declutching control. 


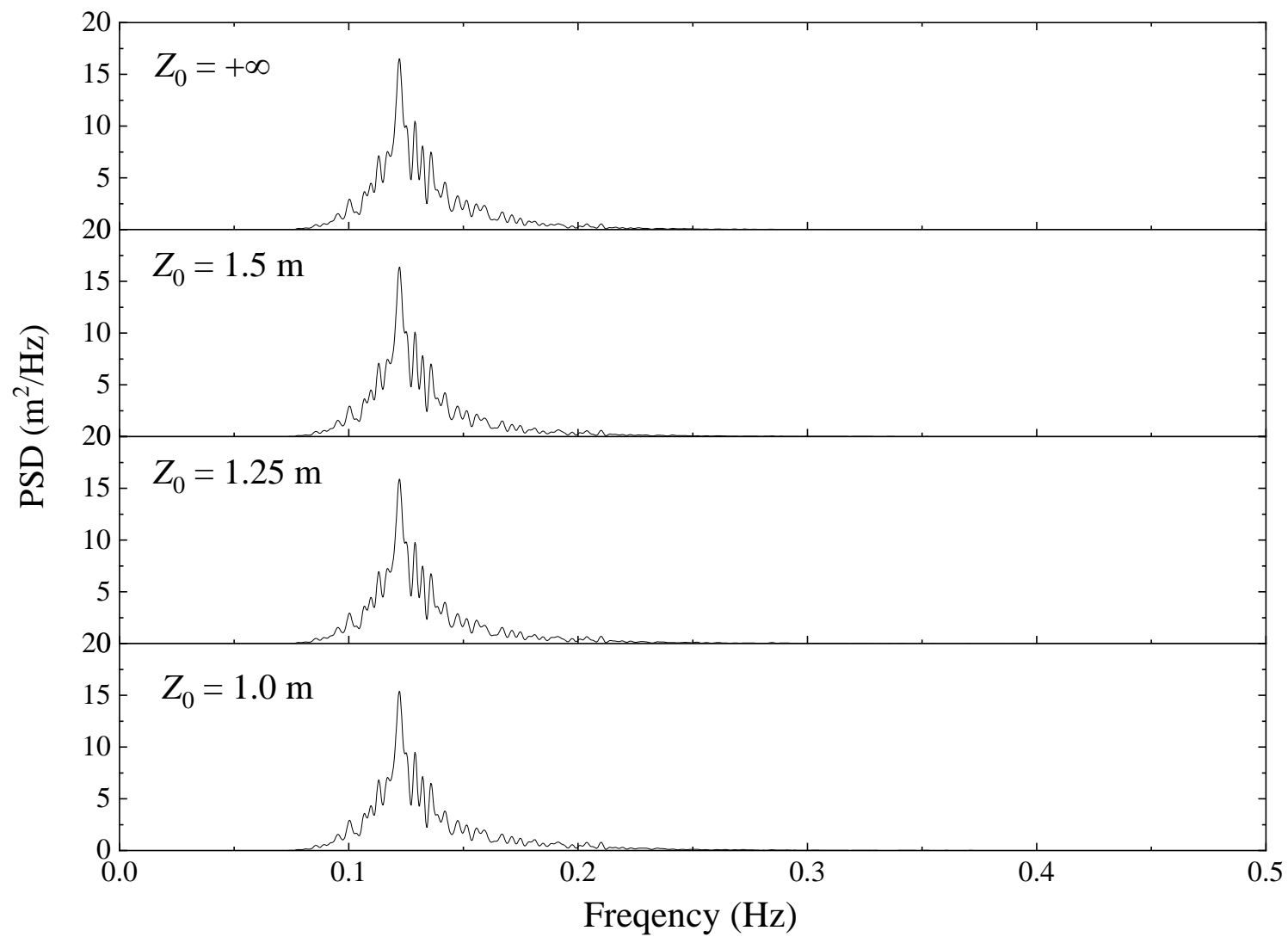

Fig. 19. PSD of external buoy motions (August).

\section{Conclusions}

A real-time wave energy control algorithm based on the model predictive control strategy is developed and implemented to a bi-oscillator WEC. To tackle the non-causality of wave energy control, artificial intelligence technology is adopted to perform online forecasting of future wave force based on free surface elevation.

Due to the implementation of the real-time control, which offloads the PTO system occasionally, the power capture efficiency is increased by about $30 \%$. The WEC oscillation is amplified as the resistance load of the PTO system is offloaded occasionally. Once the PTO is loaded back again, the WEC oscillation has been amplified resulting in the enhancement of wave power capture efficiency. Since the wave elevation rather than the wave spectra is employed in the control algorithm, it is robust to the wave condition and thus can be implemented in a wide range of sea sites. This research proposes an efficient and robust wave energy technology, leading to step change reduction in the energy cost and acceleration of the large-scale deployment of wave energy, and ultimately realizing the potential contribution of wave energy to the net zero target.

A reduced power capture efficiency has been linked to the forecasting error. The forecasted future wave load is used to optimize the control command whereas the system is 
subject to the actual wave load. Consequently, the derived control command differs from the ideal one. It is shown that the forecasting error leads to a 5\% reduction of the total energy harvested. Further analysis manifests that the energy loss is primarily attributed to the phase error.

A constraint strategy, which brakes the internal oscillator whenever its excursion exceeds a threshold, is also developed and implemented to the declutching control. Although the constraint declutching control is less efficient than the unconstraint one, it is still able to enhance the power capture of the bi-oscillator on condition that the threshold is not too low $\left(Z_{0}>1.25 \mathrm{~m}\right)$. Otherwise, the power capture could be decreased.

\section{Future Work}

The technical viability and effectiveness of the proposed control algorithm has been confirmed numerically in this work. In order to fully check its feasibility in real applications, scaled model test research in wave basin would be launched in the next stage.

\section{Acknowledgement}

The present research is supported by the State Key Laboratory of Coastal and Offshore Engineering, Dalian University of Technology (Grant No. LP1908) and State Key Laboratory of Hydraulic Engineering Simulation and Safety, Tianjin University (Grant No. HESS-1919).

\section{References}

[1] International Energy Agency. Renewables 2019-Market analysis and forecast from 2019 to 2024.2019.

[2] Parliament of the UK. The Climate Change Act 2008 (2050 Target Amendment) 2019.

[3] European Commission. Climate strategies \& targets. Available at: https://ec.europa.eu/clima/policies/strategies_en

[4] University of Edinburgh's Policy and Innovation Group Energy Systems Catapult. Wave and Tidal Energy: The Potential Economic Value. 2020.

[5] Falcão AFdO. Wave energy utilization: A review of the technologies. Renewable and Sustainable Energy Reviews. 2010;14:899-918.

[6] Falnes J. A review of wave-energy extraction. Mar Struct. 2007;20:185-201.

[7] Ning DZ, Wang RQ, Chen LF, Sun K. Experimental investigation of a land-based dualchamber OWC wave energy converter. Renew Sust Energ Rev. 2019;105:48-60.

[8] Contestabile P, Iuppa C, Di Lauro E, Cavallaro L, Andersen TL, Vicinanza D. Wave loadings acting on innovative rubble mound breakwater for overtopping wave energy conversion. Coast Eng. 2017;122:60-74. 
[9] Li L, Zhang X, Yuan Z, Gao Y. Multi-Stable Mechanism of an Oscillating-Body Wave Energy Converter. Ieee T Sustain Energ. 2020;11:500-8.

[10] Supergen Offshore Renewable Energy. Wave Energy Road Map. 2019.

[11] Falnes J. Ocean waves and oscillating systems: linear interactions including waveenergy extraction: Cambridge university press; 2002.

[12] Budal K, Falnes J. Interacting point absorbers with controlled motion, in Power from Sea Waves: BM Count, Academic Press; 1980.

[13] Henriques JCC, Gato LMC, Falcao AFO, Robles E, Fay FX. Latching control of a floating oscillating-water-column wave energy converter. Renew Energ. 2016;90:229-41.

[14] Sheng WA, Alcorn R, Lewis A. On improving wave energy conversion, part II: Development of latching control technologies. Renew Energ. 2015;75:935-44.

[15] Justino P, Falcao A. Active relief valve for an OWC wave energy devices. Proceedings of the European wave energy conference2000. p. 295-300.

[16] Babarit A, Guglielmi M, Clement AH. Declutching control of a wave energy converter. Ocean Eng. 2009;36:1015-24.

[17] Zhang XT, Yang JM, Zhao WH, Xiao LF. Effects of wave excitation force prediction deviations on the discrete control performance of an oscillating wave energy converter. Ships Offshore Struc. 2016;11:351-68.

[18] Feng Z, Kerrigan EC. Latching-Declutching Control of Wave Energy Converters Using Derivative-Free Optimization. Ieee T Sustain Energ. 2015;6:773-80.

[19] Zhang Y, Li G. Non-causal Linear Optimal Control of Wave Energy Converters with Enhanced Robustness by Sliding Mode Control. Ieee T Sustain Energ. 2019:1-.

[20] Zhan S, Li G, Bailey C. Economic Feedback Model Predictive Control of Wave Energy Converters. IEEE Transactions on Industrial Electronics. 2020;67:3932-43.

[21] Fusco F, Ringwood JV. Suboptimal Causal Reactive Control of Wave Energy Converters Using a Second Order System Model. The Twenty-first International Offshore and Polar Engineering Conference. Maui, Hawaii, USA: International Society of Offshore and Polar Engineers; 2011. p. 8.

[22] Li L, Gao Z. Maximization of wave power extraction of a heave point absorber with a sea-state-based causal control algorithm. Energy. 2020;204:117881.

[23] Liao Z, Stansby P, Li G. A generic linear non-causal optimal control framework integrated with wave excitation force prediction for multi-mode wave energy converters with application to M4. Appl Ocean Res. 2020;97:102056.

[24] Li L, Yuan Z, Gao Y, Zhang X. Wave Force Prediction Effect on the Energy Absorption of a Wave Energy Converter With Real-Time Control. Ieee T Sustain Energ. 2019;10:615-24.

[25] Cummins W. The impulse response function and ship motions. Washington DC: David Taylor Model Basin; 1962.

[26] DNV. WADAM - Wave Analysis by Diffraction and Morison Theory. SESAM user's manual, Høvik1994.

[27] Zabala I, Henriques JCC, Blanco JM, Gomez A, Gato LMC, Bidaguren I, et al. Waveinduced real-fluid effects in marine energy converters: Review and application to OWC devices. Renew Sust Energ Rev. 2019;111:535-49. 
[28] Penalba M, Giorgi G, Ringwood JV. Mathematical modelling of wave energy converters: A review of nonlinear approaches. Renew Sust Energ Rev. 2017;78:1188-207.

[29] Clement AH, Babarit A. Discrete control of resonant wave energy devices. Philos T R Soc A. 2012;370:288-314.

[30] Borne P, Dauphin-Tanguy G, Richard J, Rotella F, Zambettakis I. Méthodes et techniques de l'ingénieur, Automatique: Commande et optimisation des processus. TECNIP, Paris. 1990.

[31] Li G, Belmont MR. Model predictive control of sea wave energy converters - Part I: A convex approach for the case of a single device. Renew Energ. 2014;69:453-63.

[32] Li G, Belmont MR. Model predictive control of sea wave energy converters - Part II: The case of an array of devices. Renew Energ. 2014;68:540-9.

[33] Wu JM, Yao YX, Zhou L, Goteman M. Real-time latching control strategies for the solo Duck wave energy converter in irregular waves. Appl Energ. 2018;222:717-28.

[34] Li L, Yuan ZM, Gao Y. Maximization of energy absorption for a wave energy converter using the deep machine learning. Energy. 2018;165:340-9.

[35] Li L, Gao Z, Yuan Z-M. On the sensitivity and uncertainty of wave energy conversion with an artificial neural-network-based controller. Ocean Eng. 2019;183:282-93.

[36] Fusco F, Ringwood J. A model for the sensitivity of non-causal control of wave energy converters to wave excitation force prediction errors. Proceedings of the 9th European Wave and Tidal Energy Conference (EWTEC): School of Civil Engineering and the Environment, University of Southampton; 2011.

[37] Hong X, Billings SA. Time series multistep-ahead predictability estimation and ranking. J Forecasting. 1999;18:139-49.

[38] Wu SP, Liu CY, Chen XP. Offshore wave energy resource assessment in the East China Sea. Renew Energ. 2015;76:628-36.

[39] ASTM International. Standard Practices for Cycle Counting in Fatigue Analysis. 2017. 